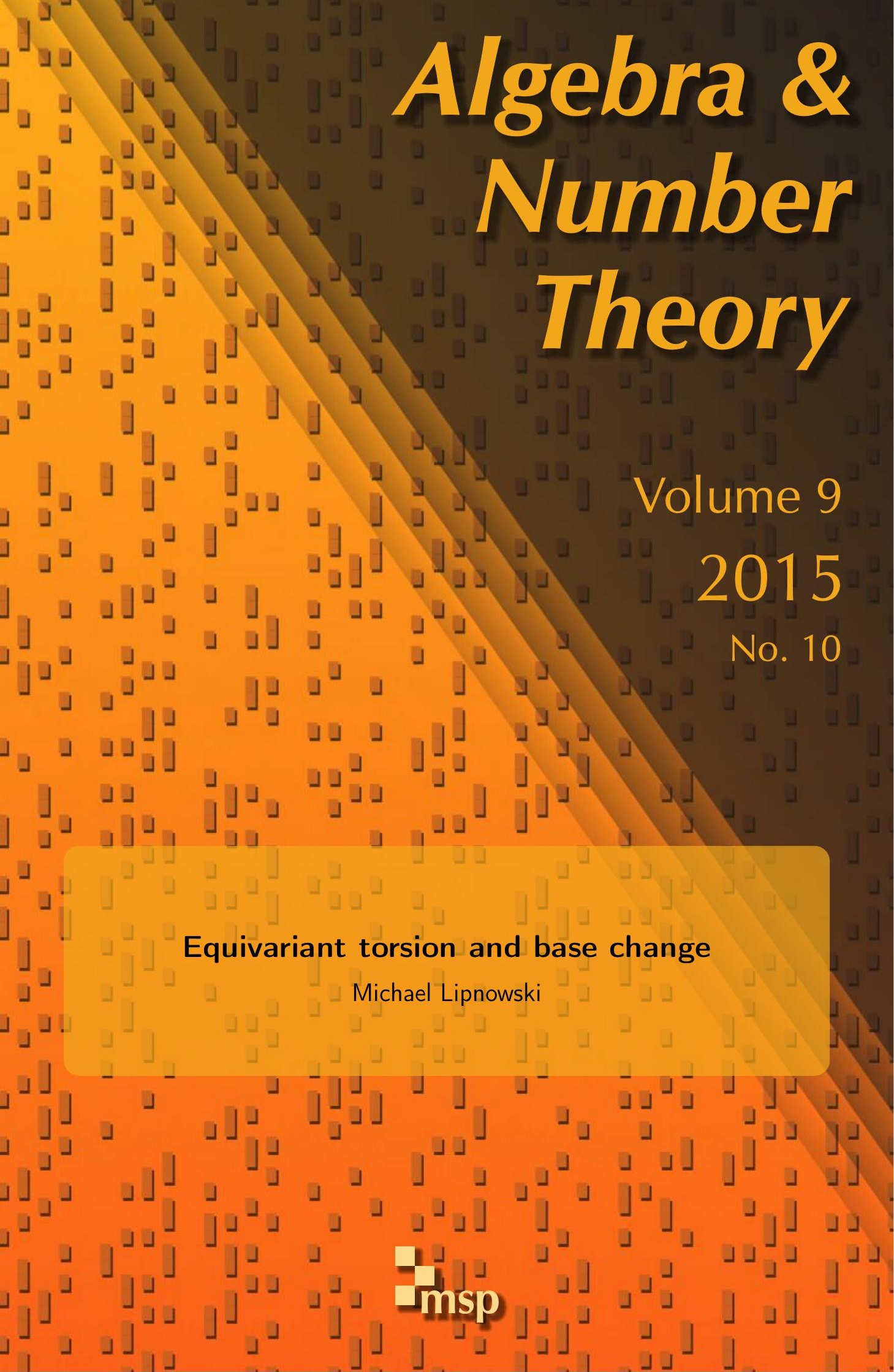




\title{
Equivariant torsion and base change
}

\author{
Michael Lipnowski
}

What is the true order of growth of torsion in the cohomology of an arithmetic group? Let $D$ be a quaternion algebra over an imaginary quadratic field $F$. Let $E / F$ be a cyclic Galois extension with $\Gamma_{E / F}=\langle\sigma\rangle$. We prove lower bounds for "the Lefschetz number of $\sigma$ acting on torsion cohomology" of certain Galoisstable arithmetic subgroups of $D_{E}^{\times}$. For these same subgroups, we unconditionally prove a would-be-numerical consequence of the existence of a hypothetical base change map for torsion cohomology.

$0 . \quad$ Introduction

1. A priori predictions via torsion base change functoriality 2203

2. Local systems over locally symmetric manifolds

3. Zeta functions and analytic torsion of equivariant, metrized local systems

4. Base change for quaternion algebras and analytic torsion

2211

5. Matching at places where $E / F$ is unramified

6. Matching at places where $E / F$ is tamely ramified

7. A numerical form of base change functoriality for torsion

8. Asymptotic growth of analytic torsion and torsion cohomology 2230

List of symbols

Acknowledgements

References

A list of symbols can be found on page 2237.

\section{Introduction}

Let $\boldsymbol{H} / \mathbb{Q}$ be a semisimple group. Let $K \subset H=\boldsymbol{H}(\mathbb{R})$ be a maximal compact subgroup and $X=X_{\boldsymbol{H}}=H / K$ the symmetric space of maximal compact subgroups of $H$. Fix an arithmetic subgroup $\Gamma \subset \boldsymbol{H}(\mathbb{Q})$. Let $\rho: \boldsymbol{H} \rightarrow \mathrm{GL}(V)$ be a homomorphism of algebraic groups over $\mathbb{Q}$ and let $M \subset V$ be a $\Gamma$-stable lattice.

MSC2010: primary 11F75; secondary 11F72, 11F70.

Keywords: torsion, cohomology, Reidemeister torsion, analytic torsion, Ray-Singer torsion, locally symmetric space, trace formula, base change, equivariant, twisted. 
Suppose that $\boldsymbol{H} / \mathbb{Q}$ is anisotropic and that $M_{\mathbb{R}}$ is strongly acyclic [Bergeron and Venkatesh 2013, §4]. Let $\Gamma_{n} \subset \Gamma$ be a sequence of subgroups for which the injectivity radius of $\Gamma_{n} \backslash X$ approaches infinity. Bergeron and Venkatesh [2013] prove that if the fundamental $\operatorname{rank}^{1}$ of $X$ equals 1 , then

$$
\liminf _{n} \frac{\sum_{i} \log \left|H^{i}\left(\Gamma_{n}, M\right)_{\text {tors }}\right|}{\left[\Gamma: \Gamma_{n}\right]}>0
$$

Little is known about the true order of growth of $\log \left|H^{*}\left(\Gamma_{n}, M\right)_{\text {tors }}\right|$ for $X$ of fundamental rank $\neq 1$.

Let $F$ be an imaginary quadratic field and let $E / F$ be a cyclic Galois extension of degree $p$. Let $D$ be a quaternion algebra over $F$. One goal of this paper is to prove lower bounds for the amount of torsion in the cohomology of locally symmetric spaces for $X_{\mathrm{PGL}_{1}\left(D_{E}\right)}$, which has fundamental rank $p>1$.

Calegari and Venkatesh [2012, §2] have proposed an analogue of Langlands functoriality for torsion cohomology. The hypothetical existence of torsion base change functoriality leads one to predict that torsion cohomology on locally symmetric spaces for $X_{\mathrm{PGL}_{1}(D)}$ - proven to be abundant in [Bergeron and Venkatesh 2013] can be lifted to torsion cohomology on locally symmetric spaces for $X_{\mathrm{PGL}_{1}\left(D_{E}\right)}$. A second goal of this paper is to unconditionally prove a numerical relationship between the cohomology of certain locally symmetric spaces for $X_{\mathrm{PGL}_{1}\left(D_{E}\right)}$ and "matching" locally symmetric spaces for $X_{\mathrm{PGL}_{1}(D)}$ which is consistent with base change for torsion.

\section{A. Notational setup for statement of main results.}

0A1. Analytic torsion and Reidemeister torsion. Let $\mathcal{L} \rightarrow \mathcal{M}$ be a local system of $\mathbb{C}$-vector spaces, equipped with a Hermitian metric, over a compact Riemannian manifold $\mathcal{M}$. Let $\sigma$, of prime order $p$, act equivariantly by isometries on $\mathcal{L} \rightarrow \mathcal{M}$. Let $\Delta_{\mathcal{L}}$ denote the Laplace operator for $\mathcal{L}$. Let $\tau_{\sigma}(\mathcal{M}, \mathcal{L})$ denote the $\sigma$-equivariant analytic torsion of $\mathcal{L} \rightarrow \mathcal{M}$; it is a spectral quantity defined purely in terms of the action of $\sigma$ on the eigenspaces of $\Delta_{\mathcal{L}}$ (see Section 3A).

We define the untwisted analytic torsion of a metrized local system $L \rightarrow M$ to be $\tau(M, L):=\tau_{1}(M, L)$.

We let $\mathrm{RT}_{\sigma}(\mathcal{M}, \mathcal{L})$ denote the equivariant Reidemeister torsion of $\mathcal{L} \rightarrow \mathcal{M}$; it is an equivariant topological invariant, described in a manner tailored to our needs in [Lipnowski 2014, §1.3].

We let $\mathrm{RT}(M, L):=\mathrm{RT}_{1}(M, L)$ denote untwisted Reidemeister torsion.

${ }^{1}$ The fundamental rank of $H / K$ is $\operatorname{rank}(H)-\operatorname{rank}(K)$, where rank denotes the dimension of any maximal torus, not necessarily split. 
0A2. The locally symmetric spaces. Let $D$ be a quaternion algebra over an imaginary quadratic field $F$. Let $E / F$ be a cyclic Galois extension of prime degree $p$, with Galois group $\Gamma_{E / F}$; we fix a generator $\sigma$ for $\Gamma_{E / F}$. Let $\boldsymbol{G}$ denote the adjoint group of $\underline{D}^{\times}$, the unit group of $D$ considered as an $F$-algebraic group. We form the associated locally symmetric space

$$
\mathcal{M}_{\mathcal{U}}=\boldsymbol{G}(E) \backslash \boldsymbol{G}\left(\mathbb{A}_{E}\right) / \mathcal{K U}, \quad M_{U}=\boldsymbol{G}(F) \backslash \boldsymbol{G}\left(\mathbb{A}_{F}\right) / K U,
$$

where $\mathcal{U}$ is a compact open Galois-stable subgroup of $\boldsymbol{G}\left(\mathbb{A}_{E}^{\mathrm{fin}}\right) ; U$ is a compact open subgroup of $\boldsymbol{G}\left(\mathbb{A}_{F}^{\mathrm{fin}}\right) ; \mathcal{K}$ is a Galois-stable maximal compact subgroup of $\boldsymbol{G}\left(E_{\mathbb{R}}\right)$; and $K=\mathcal{K}^{\Gamma_{E / F}}$ is a maximal compact subgroup of $\boldsymbol{G}\left(F_{\mathbb{R}}\right)$. There is a $\boldsymbol{G}\left(E_{\mathbb{R}}\right) \rtimes \Gamma_{E / F^{-}}$ invariant metric on $\boldsymbol{G}\left(E_{\mathbb{R}}\right) / \mathcal{K}$ and a $\boldsymbol{G}\left(F_{\mathbb{R}}\right)$-invariant metric on $\boldsymbol{G}\left(F_{\mathbb{R}}\right) / K$ which descend to metrics on $\mathcal{M}_{\mathcal{U}}$ and $M_{U}$ respectively; these invariant metrics are unique up to scaling. For a discussion of normalization of these metrics, see Section 2.

0A3. The local systems. Let $N / F$ be a finite extension and $V$ an $N$-vector space. Let $\tilde{\rho}: \operatorname{Res}_{E / F} \boldsymbol{G} \rtimes \Gamma_{E / F} \rightarrow \operatorname{Res}_{N / F} \operatorname{GL}(V)$ and $\rho: \boldsymbol{G} \rightarrow \operatorname{Res}_{N / F} \operatorname{GL}(W)$ be algebraic representations (over $F$ ). We fix "integral structures" within $V$ and $W$, i.e., $O_{N}$-lattices inside $V$ and $W$; let $\mathcal{U}_{0}$ and $U_{0}$ be their respective stabilizers inside $\boldsymbol{G}\left(\mathbb{A}_{E}^{\text {fin }}\right)$ and $\boldsymbol{G}\left(\mathbb{A}_{F}^{\mathrm{fin}}\right)$.

The representation $\tilde{\rho}$ gives rise to a local system of $N$-vector spaces $\mathcal{L}_{\tilde{\rho}} \rightarrow \mathcal{M}_{\mathcal{U}}$ with an action of $\Gamma_{E / F}$. Similarly, the representation $\rho$ gives rise to a local system of $N$-vector spaces $L_{\rho} \rightarrow M_{U}$. Let $\mathcal{L}_{\tilde{\rho}_{l}}, L_{\rho_{l}}$ denote the scalar extensions of $\mathcal{L}_{\tilde{\rho}}, L_{\rho}$ by the complex embedding $\iota: N \hookrightarrow \mathbb{C}$. If $\mathcal{U} \subset \mathcal{U}_{0}$ and $U \subset U_{0}$, the integral structures on $V, W$ yield integral structures on these local systems, i.e., local systems of $O_{N}$-modules, which we denote by

$$
\mathcal{L}_{\tilde{\rho}}^{0} \rightarrow \mathcal{M}_{\mathcal{U}}, \quad L_{\rho}^{0} \rightarrow M_{U}
$$

We discuss the integral structures and scalar extensions in greater detail in Section 2B.

0B. Statements of main results. Let

$$
M_{U}=\boldsymbol{G}(F) \backslash \boldsymbol{G}\left(\mathbb{A}_{F}\right) / K U, \quad \text { where } K=\mathcal{K} \cap \boldsymbol{G}\left(F_{\mathbb{R}}\right), U=\mathcal{U} \cap \boldsymbol{G}\left(\mathbb{A}_{F}^{\mathrm{fin}}\right) .
$$

Sample Theorem (comparison of analytic torsion). Let $E / F$ be an everywhere unramified Galois extension of odd prime degree. Suppose that $\mathcal{U}$ is a parahoric level structure (see Definition 5.1) and that $\rho$ and $\tilde{\rho}$ match (see Section 4C). Then, for any complex embedding $\iota: N \hookrightarrow \mathbb{C}$,

$$
\tau_{\sigma}\left(\mathcal{M}_{\mathcal{U}}, \mathcal{L}_{\tilde{\rho}_{l}}\right)=\tau\left(M_{U}, L_{\rho_{l}}\right)^{p} .
$$

A more general statement, which allows any $E / F$ that is everywhere tamely ramified, is proven in Sections $4 \mathrm{D}$ and 6. The relationship in the more general case between $\tau_{\sigma}\left(\mathcal{M}_{\mathcal{U}}, \mathcal{L}_{\tilde{\rho}_{l}}\right)$ and $\tau\left(M_{U}, L_{\rho_{l}}\right)$ has the same flavor but is not as simply stated. 
Spectral comparisons such as the sample theorem, in conjunction with CheegerMüller theorems (see Section 0C), have consequences for torsion in the cohomology of $\mathcal{L}_{\tilde{\rho}}^{0}$. In order to describe these implications, we use the following notational shorthand:

- $\sum^{*}$ denotes an alternating sum.

- $P$ denotes the $p$-cyclotomic polynomial $P(x)=x^{p-1}+x^{p-2}+\cdots+1$.

- For any $\mathbb{Z}[\sigma]$-module $A$ and any polynomial $h \in \mathbb{Z}[x]$, we let $A^{h(\sigma)}$ be the set $\{a \in A: h(\sigma) a=0\}$.

Sample Theorem (relationship between sizes of torsion subgroups). Let $E / F$ be an everywhere tamely ramified Galois extension of odd prime degree with $\Gamma_{E / F}=\langle\sigma\rangle$. Let the places where $E / F$ is ramified and the places where $D$ is ramified be disjoint. Suppose that:

- $\rho, \tilde{\rho}$ are matching representations of the sort described in Section 7A.

- The level structure $\mathcal{U}$ is tamely parahoric at each unramified place of $E / F$ (see Definition 6.16).

Then there is an explicit finite collection of compact open subgroups $U \subset \boldsymbol{G}\left(\mathbb{A}_{F}^{\mathrm{fin}}\right)$ and explicit constants $c_{U}$ such that

$$
\begin{aligned}
\sum^{*} \log \left|H^{i}\left(\mathcal{M}_{\mathcal{U}}, \mathcal{L}_{\tilde{\rho}}^{0}\right)_{\text {tors }}^{\sigma-1}\right|-\frac{1}{p-1} \log \left|H^{i}\left(\mathcal{M}_{\mathcal{U}}, \mathcal{L}_{\tilde{\rho}}^{0}\right)_{\text {tors }}^{P(\sigma)}\right| \\
=\sum_{U} c_{U} \sum^{*} \log \left|H^{i}\left(M_{U}, L_{\rho}^{0}\right)_{\text {tors }}\right|+n \log p
\end{aligned}
$$

for some integer $n$. Furthermore, $n$ can be bounded linearly by $\operatorname{dim}_{\mathbb{F}_{p}} H^{i}\left(\mathcal{M}_{\mathcal{U}}^{\sigma}, \mathcal{L}_{\rho, \mathbb{F}_{p}}^{0}\right)$.

For a more precise statement, see Theorem 7.4. An appropriate generalization of the sample theorem also has consequences for growth of torsion in the cohomology of the spaces $\mathcal{M}_{\mathcal{U}}$.

Corollary (growth of torsion for fundamental rank $>1$ ). Let $E / F$ be everywhere tamely ramified with $[E: F]$ odd. Let the places where $E / F$ is ramified and the places where $D$ is ramified be disjoint. Let $\mathcal{U}_{n} \subset \mathcal{U}_{0}$ denote a sequence of compact open subgroups of $\boldsymbol{G}\left(\mathbb{A}_{E}^{\text {fin }}\right)$ such that:

- The injectivity radius of $\mathcal{M}_{\mathcal{U}_{n}}$ approaches $\infty$.

- The level structures $\mathcal{U}_{n}$ are tamely parahoric at each unramified place of $E / F$ (see Definition 6.16).

- Let $U_{n} \subset \boldsymbol{G}\left(\mathbb{A}_{F}^{\mathrm{fin}}\right)$ denote the matching level structure implicit in the definition of tamely parahoric (see Definition 6.16). For every complex embedding $\iota: N \hookrightarrow \mathbb{C}$, the local systems $L_{\rho_{l}} \rightarrow M_{U_{n}}$ form a strongly acyclic family (see Section 8A), where $\rho$ and $\tilde{\rho}$ are matching representations (see Section $4 \mathrm{C}$ ). 
Then

$$
\limsup _{n} \frac{\log \left|H^{*}\left(\mathcal{M}_{\mathcal{U}_{n}}, \mathcal{L}_{\tilde{\rho}}^{0}\right)\right|}{\operatorname{vol}\left(\mathcal{M}_{\mathcal{U}_{n}}\right)^{1 / p}}>0 .
$$

In fact, we prove an asymptotic formula for the "Lefschetz numbers of $\sigma$ acting on $H^{*}\left(\mathcal{M}_{\mathcal{U}_{n}}, \mathcal{L}_{\tilde{\rho}}^{0}\right)_{\text {tors }}$ " (see Section $8 \mathrm{C}$ ).

0C. Main tools. Three main inputs are used to prove these theorems:

(a) Cheeger-Müller theorems. Let $\mathcal{L} \rightarrow \mathcal{M}$ be a local system of metrized $\mathbb{C}$-vector spaces acted on equivariantly by an isometry $\sigma$ of finite order $p$. A Cheeger-Müller theorem is an identity

$$
\tau_{\sigma}(\mathcal{M}, \mathcal{L})=\mathrm{RT}_{\sigma}(\mathcal{M}, \mathcal{L})
$$

valid for some class of metrized local systems $\mathcal{L}$ and some class of equivariant isometries $\sigma$. This remarkable formula was originally fathomed, for $\sigma=1$ and $\mathcal{L}$ unitarily flat, by Ray and Singer [1971]. When $\sigma$ equals 1 and $\mathcal{L}$ is unitarily flat, it was proven independently by Cheeger [1979] and Müller [1978]. An extension to $\sigma=1$ and general unimodular $\mathcal{L}$ was proven by Müller [1993].

A general version of this theorem for $\sigma \neq 1$ is proven by Bismut and Zhang [1994]. This general version expresses the difference between $\log \operatorname{RT}_{\sigma}(\mathcal{M}, \mathcal{L})$ and $\log \tau_{\sigma}(\mathcal{M}, \mathcal{L})$ in terms of auxiliary differential geometric data on a germ of the fixed point set of $\sigma$. The author proves in [Lipnowski 2014] that this difference equals zero in the cases to be studied in this paper.

Cheeger-Müller theorems provide a bridge between the analytic expression $\tau_{\sigma}(\mathcal{M}, \mathcal{L})$ and the quantity $\operatorname{RT}_{\sigma}(\mathcal{M}, \mathcal{L})$. In the case where $\mathcal{L}$ is the complexification of a local system of $\mathbb{Z}$-modules $\mathcal{L}^{0}$, the latter can concretely be related to the $\sigma$-module $H^{*}\left(\mathcal{M}, \mathcal{L}^{0}\right)$ tors [Lipnowski 2014, Corollary 3.8].

(b) Trace formula comparison. Using Cheeger-Müller theorems, torsion in cohomology of arithmetic groups related by base change can be compared by instead comparing analytic torsions. In the case of compact quotient, the equivariant analytic torsion of $(\mathcal{M}, \mathcal{L})$ is determined by the spectral side of the twisted Arthur-Selberg trace formula for an appropriate family of test functions (see Section 3). The analytic torsion of $(M, L)$ is determined by the spectral side of the untwisted trace formula for a matching family of test functions.

Comparing these spectral quantities uses the methods of [Langlands 1980] together with some local representation theory for $\mathrm{PGL}_{2}$. In particular, we need to prove a "fundamental lemma for the spherical unit" for tamely ramified base change of $\mathrm{PGL}_{2}$.

(c) The results of Bergeron and Venkatesh [2013] on growth of untwisted analytic torsion for sequences of locally symmetric spaces with universal cover of fundamental rank 1 . 
Combining (a) with (b) proves an identity relating sizes of torsion cohomology for arithmetic groups related by base change (see Theorem 7.4). The resulting identity is consistent with implications of torsion base change functoriality (see Section 1).

Combining (a) with (c) proves the growth of "Lefschetz numbers for torsion". See Theorem 8.5 for a more precise statement.

0D. Outline. In Section 1, we discuss base change functoriality over $\mathbb{Z}$. Base change functoriality over $\mathbb{Z}$ predicts one of the main results of this paper, a relationship between the sizes of torsion subgroups on locally symmetric spaces related by base change.

In Section 2, we discuss (equivariant) local systems over locally symmetric spaces. We explain in Section 2B how, for finite extensions $N / F$, algebraic homomorphisms $\boldsymbol{G} \rightarrow R_{N / F} \mathrm{GL}(V)$ over $F$ give rise to local systems of $O_{N}$-modules over $M_{U}$ for appropriate compact open subgroups $U \subset \boldsymbol{G}\left(\mathbb{A}_{F}^{\mathrm{fin}}\right)$.

In Section 3, we express the $\sigma$-twisted analytic torsion of $\mathcal{L}_{\tilde{\rho}_{t}} \rightarrow \mathcal{M}_{\mathcal{U}}$ and the untwisted analytic torsion of $L_{\rho_{l}} \rightarrow M_{U}$ in purely representation theoretic terms.

In Section 4, we use Langlands' representation theoretic statement of base change to prove an abstract matching Section 4D, which will ultimately imply identities of the flavor

$$
\tau_{\sigma}\left(\mathcal{M}_{\mathcal{U}}, \mathcal{L}_{\tilde{\rho}_{l}}\right)=\tau\left(M_{U}, L_{\rho_{l}}\right)^{p}
$$

of the aforementioned sample theorem. In order to apply this matching theorem, we need to find instances of matching test functions, which will be the objective of Sections 5 and 6.

In Section 5, we describe some circumstances under which the desired matching test functions can be found. This matching only applies at places $v$ where $E_{v} / F_{v}$ is unramified. Here is where we finally define and discuss parahoric level structure.

In Section 6, we prove a matching theorem at places $v$ where $E_{v} / F_{v}$ is tamely ramified. In Section 6D, we define tamely parahoric level structures, those level structures which occur in the matching theorem (Theorem 6.17) and the numerical cohomology comparison theorem (Theorem 7.4).

In Section 7, we prove the numerical cohomology comparison theorem (Theorem 7.4) for the local systems introduced in Section 7A.

In Section 8, we use the main comparison corollary for analytic torsion (Corollary 4.19) together with [Bergeron and Venkatesh 2013, Theorem 4.5] to prove that, for appropriate equivariant local systems $\mathcal{L}$ and level structures $\mathcal{U}$ (see Definition 5.1), the twisted analytic torsion $\log \tau_{\sigma}\left(\mathcal{M}_{\mathcal{U}}, \mathcal{L}\right)$ is asymptotic to $\operatorname{vol}\left(\mathcal{M}_{\mathcal{U}}\right)^{1 / p}$. Combined with the results of [Lipnowski 2014, $\S \S 1-5]$ — which relate equivariant Reidemeister torsion to cohomology - asymptotic growth of cohomology is proven. The results of [Bergeron and Venkatesh 2013] prove asymptotic growth of Reidemeister 
torsion, which one might loosely think of as an "Euler characteristic for torsion in cohomology". In the same vein, the results of Section 8 prove "asymptotic growth of Lefschetz numbers for torsion in cohomology".

\section{A priori predictions via torsion base change functoriality}

Calegari and Venkatesh [2012, §2] have conjectured an analogue of Langlands functoriality for mod $p$ and torsion cohomology of arithmetic locally symmetric spaces. In this section, we explain how one of our main results, Theorem 7.4, is roughly predicted by their conjecture applied to base change.

1A. Base change functoriality over $\mathbb{Z}$. For a more general discussion of functoriality over $\mathbb{Z}$, we refer the reader to [Calegari and Venkatesh 2012, §2].

Let $F$ be any number field and $E / F$ a cyclic Galois extension of odd prime degree $p$. Let $\boldsymbol{G}_{1} / F$ be any group and let $\boldsymbol{G}_{2}=R_{E / F} \boldsymbol{G}_{1}$. In accordance with functoriality over $\mathbb{Z}$, the diagonal map of $L$-groups [Buzzard and Gee 2015]

$$
{ }^{L} \boldsymbol{G}_{1} \stackrel{\phi}{\longrightarrow}{ }^{L} \boldsymbol{G}_{2}, \quad(g, \sigma) \mapsto(g, \ldots, g) \times \sigma
$$

is expected to give rise to a Hecke-equivariant map $\phi_{*}$ on cohomology, torsion or otherwise. For certain groups such as $\boldsymbol{G}_{1}$, the unit group of a semisimple algebra over $F$, this correspondence is known for characteristic zero cohomology.

One main purpose of this paper is to unconditionally prove certain would-benumerical consequences of the existence of the hypothetical map $\phi_{*}$. The analogue of this program was carried out for Jacquet-Langlands functoriality for $\mathrm{PGL}_{2}$ in [Calegari and Venkatesh 2012].

1B. Conjectures on torsion base change. This highly speculative section discusses implications of the existence of a base change map $\phi_{*}$, as above. The discussion uses the language of Langlands' theory of base change, to be reviewed in Section 4A.

Let $D$ be a quaternion algebra over a number field $F$. Let $\boldsymbol{G}$ denote the adjoint group of its group of units. Let $E / F$ be a cyclic Galois extension with $\Gamma_{E / F}=\langle\sigma\rangle$. Let $\mathcal{U} \subset \boldsymbol{G}\left(\mathbb{A}_{E}^{\text {fin }}\right)$ and $U \subset \boldsymbol{G}\left(\mathbb{A}_{F}^{\mathrm{fin}}\right)$ be compact open subgroups with $\mathcal{U}$ Galoisinvariant. Let $\mathcal{U}$ and $U$ have respective volume-1 Haar measures $d \tilde{u}$ and $d u$. Let $K \subset \boldsymbol{G}\left(F_{\mathbb{R}}\right)$ be a maximal compact subgroup and $\mathcal{K} \subset \boldsymbol{G}\left(E_{\mathbb{R}}\right)$ a Galois-invariant maximal compact subgroup. Let

$$
\mathcal{M}_{\mathcal{U}}=\boldsymbol{G}(E) \backslash \boldsymbol{G}\left(\mathbb{A}_{E}\right) / \mathcal{K U} \quad \text { and } \quad M_{U}=\boldsymbol{G}(F) \backslash \boldsymbol{G}\left(\mathbb{A}_{F}\right) / K U .
$$

For compact open subgroups $J \subset \boldsymbol{G}\left(\mathbb{A}_{F}^{\text {fin }}\right)$ and $\mathcal{J} \subset \boldsymbol{G}\left(\mathbb{A}_{E}^{\text {fin }}\right)$, let

$$
W^{J}=L^{2}\left(\boldsymbol{G}(F) \backslash \boldsymbol{G}\left(\mathbb{A}_{F}\right) / J\right) \quad \text { and } \quad \mathcal{W}^{\mathcal{J}}=L^{2}\left(\boldsymbol{G}(E) \backslash \boldsymbol{G}\left(\mathbb{A}_{E}\right) / \mathcal{J}\right) .
$$


Definition 1.1. We say that $\mathbf{1}_{\mathcal{U}} d \tilde{u}$ and $\sum_{U} c_{U} \mathbf{1}_{U} d u$ (finite sum) are matching level structures if

$$
\operatorname{tr}\left\{\sigma \mid \tilde{\pi}_{\text {fin }}^{\mathcal{U}}\right\}=\sum_{U} c_{U} \operatorname{dim} \pi_{\text {fin }}^{U}
$$

for every pair of representations $\tilde{\pi}$ of $\boldsymbol{G}\left(\mathbb{A}_{E}\right) \rtimes \Gamma_{E / F}$ and $\pi$ of $\boldsymbol{G}\left(\mathbb{A}_{F}\right)$ which match by base change (see Section $4 \mathrm{~A}$ ). If $E^{\prime} / F$ is a second cyclic Galois extension and $\mathcal{U}^{\prime} \subset \boldsymbol{G}\left(\mathbb{A}_{E^{\prime}}\right)$ and $\mathcal{U} \subset \boldsymbol{G}\left(\mathbb{A}_{E}\right)$ both match a common level structure, we say that $\mathcal{U}$ and $\mathcal{U}^{\prime}$ are related.

Lemma 1.2. Matching level structures satisfy the identity of traces in cohomology

$$
\operatorname{tr}\left\{\sigma \mid H^{*}\left(\mathcal{M}_{\mathcal{U}}, \mathcal{L}_{\mathbb{C}}\right)\right\}=\sum_{U} c_{U} \operatorname{dim} H^{*}\left(M_{U}, L_{\mathbb{C}}\right)
$$

for local systems $\mathcal{L}, L$ associated to compatible representations $\tilde{\rho}$ of $R_{E / F} \boldsymbol{G} \rtimes \Gamma_{E / F}$ and $\rho$ of $\boldsymbol{G}$ (see Section $4 \mathrm{C}$ for a discussion of matching representations and matching local systems).

Proof. We can decompose

$$
H^{*}\left(M_{U}, L_{\mathbb{C}}\right)=\bigoplus \operatorname{dim} \operatorname{Hom}_{\boldsymbol{G}\left(\mathbb{A}_{F}\right)}(\pi, W) \operatorname{dim} \pi_{\text {fin }}^{U} \cdot H^{*}\left(\pi_{\infty} \otimes \rho\right)
$$

in accordance with Matsushima's formula [Borel and Wallach 2000, Chapter VII, Theorem 5.2], where $H^{*}\left(\pi_{\infty} \otimes \rho\right)$ denotes $(\mathfrak{g}, K)$-cohomology; this is a representation theoretic incarnation of Hodge theory. Because the level structures match, we are reduced to proving that

$$
\operatorname{tr}\left\{\sigma \mid H^{*}\left(\tilde{\pi}_{\infty} \otimes \tilde{\rho}\right)\right\}=\operatorname{dim} H^{*}\left(\pi_{\infty} \otimes \rho\right)
$$

for pairs of representations $\tilde{\pi}$ of $\boldsymbol{G}\left(\mathbb{A}_{E}\right) \rtimes \Gamma_{E / F}$ and $\pi$ of $\boldsymbol{G}\left(\mathbb{A}_{F}\right)$ which match by local base change. In this particular situation, $\boldsymbol{G}\left(E_{\mathbb{R}}\right)$ is isomorphic to $\boldsymbol{G}\left(F_{\mathbb{R}}\right)^{p}$, and $\tilde{\pi}_{\infty} \cong \pi_{\infty}^{\otimes p}$, where $\sigma$ acts by cyclic permutation. By the Künneth formula for $(\mathfrak{g}, K)$-cohomology, $H^{*}\left(\tilde{\pi}_{\infty} \otimes \tilde{\rho}\right) \cong H^{*}\left(\pi_{\infty} \otimes \rho\right)^{\otimes p}$ (graded tensor product), where $\sigma$ again acts by cyclic permutation. The result then follows by the elementary fact that, for any finite dimensional $V$,

$$
\operatorname{tr}\left\{\text { cyclic permutation } \mid V^{\otimes p}\right\}=\operatorname{dim} V .
$$

Corollary 1.3. If $\mathcal{U} \subset \boldsymbol{G}\left(\mathbb{A}_{E}\right)$ and $\mathcal{U}^{\prime} \subset \boldsymbol{G}\left(\mathbb{A}_{E^{\prime}}\right)$ are related level structures, then

$$
\operatorname{tr}\left\{\sigma \mid H^{*}\left(\mathcal{M}_{\mathcal{U}}, \mathcal{L}_{\mathbb{C}}\right)\right\}=\operatorname{tr}\left\{\sigma \mid H^{*}\left(\mathcal{M}_{\mathcal{U}^{\prime}}^{\prime}, \mathcal{L}_{\mathbb{C}}^{\prime}\right)\right\} .
$$

We optimistically conjecture that an analogous conjecture is true of torsion cohomology. 
Conjecture 1.4 (Galois structure). For related rationally acyclic local systems $\mathcal{L}^{0} \rightarrow \mathcal{M}_{\mathcal{U}}, \mathcal{L}^{\prime 0} \rightarrow \mathcal{M}_{\mathcal{U}^{\prime}}$, the graded $\mathbb{Z}[\sigma]$-modules $H^{*}\left(\mathcal{M}_{\mathcal{U}^{\prime}}^{\prime}, \mathcal{L}^{\prime 0}\right)$ and $H^{*}\left(\mathcal{M}_{\mathcal{U}}, \mathcal{L}^{0}\right)$ are isomorphic.

Let $E^{\prime}=F \times \cdots \times F / F$ be a split extension of degree $p$ and $E / F$ a cyclic Galois extension of degree $p$. For simplicity, suppose that there are compact open subgroups $\mathcal{U} \subset \boldsymbol{G}\left(\mathbb{A}_{E}^{\mathrm{fin}}\right), \quad J \subset \boldsymbol{G}\left(\mathbb{A}_{F}^{\mathrm{fin}}\right)$ for which $\mathbf{1}_{\mathcal{U}} d u$ matches $\mathbf{1}_{J} d j$; examples of this sort are constructed in Section 5. If we simply take $\mathcal{U}^{\prime}=J \times \cdots \times J$, then $\mathcal{U}$ and $\mathcal{U}^{\prime}$ are related and we have $\mathcal{M}_{\mathcal{U}^{\prime}}^{\prime}=M_{J} \times \cdots \times M_{J}$. Suppose that $\mathcal{L}^{0} \rightarrow \mathcal{M}_{\mathcal{U}}$ and $L^{0} \rightarrow M_{J}$ are matching (rationally acyclic) local systems (see Section $4 \mathrm{C}$ ). Then Conjecture 1.4 predicts that

$$
\begin{aligned}
H^{*}\left(\mathcal{M}_{\mathcal{U}}, \mathcal{L}^{0}\right) \cong_{\mathbb{Z}[\sigma]} H^{*}\left(\mathcal{M}_{\mathcal{U}^{\prime}}^{\prime},\right. & \left.\left(L^{0}\right)^{\bigotimes p}\right) \\
& =H^{*}\left(M_{J} \times \cdots \times M_{J},\left(L^{0}\right)^{\bigotimes p}\right) \cong\left(H^{*}\left(M_{J}, L^{0}\right)\right)^{\widehat{\otimes} p},
\end{aligned}
$$

where $\widehat{\otimes} p$ denotes the $p$-fold left-derived tensor product. For example, suppose the $\ell$-primary part of $H^{*}\left(M_{J}, L^{0}\right)$ is isomorphic to $\mathbb{Z} / \ell \mathbb{Z}$ in degree $d$, generated by $c \in H^{d}\left(M_{J}, L^{0}\right)$. Conjecture 1.4 predicts the existence of a graded $\mathbb{Z}[\sigma]$-submodule $\widetilde{C} \subset H^{*}\left(\mathcal{M}_{\mathcal{U}}, \mathcal{L}^{0}\right)$ isomorphic to $H^{*}\left((\mathbb{Z} \stackrel{\ell}{\longrightarrow} \mathbb{Z})[d]^{\otimes p}\right)$; the action of $\sigma$ on the latter group is induced by cyclic permutation of the tensor factors.

Remark 1.5. One computes that $H^{*}\left((\mathbb{Z} \stackrel{\ell}{\longrightarrow} \mathbb{Z})[d]^{\otimes p}\right)$ is isomorphic, as a graded $\mathbb{Z}[\sigma]$-module, to the exterior algebra on the $\mathbb{Z} / \ell \mathbb{Z}$-vector space

$$
(\mathbb{Z} / \ell \mathbb{Z})[\sigma] /\left\langle\sigma^{p-1}+\cdots+\sigma+1\right\rangle
$$

starting in degree $p(d-1)+1$.

We mention, in passing, an even more speculative conjecture, pertaining to Hecke-equivariance of this correspondence on cohomology.

Conjecture 1.6 (Hecke-equivariance of base change). Suppose $c, \widetilde{C}$ are as above. Suppose that c is a $\boldsymbol{G}$-Hecke eigenclass, i.e., for every $v$ where $\boldsymbol{G} / F_{v}$ is split and $J_{v}$ is hyperspecial and for every $\rho \in \operatorname{Rep}\left({ }^{L} \boldsymbol{G}\right)$,

$$
T_{\rho}(c)=a_{\rho} c
$$

here, $T_{\rho} \in \mathcal{H}_{v}$ denotes the image of $\rho$ under the Satake isomorphism $\mathcal{H}_{v} \cong \operatorname{Rep}\left({ }^{L} \boldsymbol{G}\right)$. Then, for every $\tilde{c} \in \widetilde{C}$ and every $\tau \in \operatorname{Rep}\left({ }^{L} R_{E / F} G\right)$,

$$
T_{\tau} \tilde{c}=a_{\tau \circ \phi} \tilde{c} .
$$

1C. A numerical consequence to be expected of torsion base change. Let $\mathcal{U}, \mathcal{U}^{\prime}$, $\mathcal{L}, L, E, E^{\prime}, F$ be as in the previous section. 
For Galois-equivariant, rationally acyclic local systems $\mathcal{L}^{0} \rightarrow \mathcal{M}_{\mathcal{U}}$ as above, where $\Gamma_{E / F}=\langle\sigma\rangle$, we consider the alternating product

$$
R_{\sigma}(\mathcal{L}):=\prod^{*} \frac{\left|H^{i}\left(\mathcal{M}_{\mathcal{U}}, \mathcal{L}^{0}\right)^{\sigma-1}\right|}{\left|H^{i}\left(\mathcal{M}_{\mathcal{U}}, \mathcal{L}^{0}\right)^{P(\sigma)}\right|^{\frac{1}{p-1}}}
$$

In support of Conjecture 1.4 and Remark 1.5, we compute in [Lipnowski 2014, Lemma 5.3] that

$$
R_{\sigma}(\mathcal{L})=R_{\sigma}\left(L^{\otimes p}\right)=R(L)^{p}, \quad R(L):=\prod^{*}\left|H^{i}\left(M_{J}, L^{0}\right)\right|,
$$

at least up to powers of $p$.

The invariant $R_{\sigma}(\mathcal{L})$ is very closely related to the twisted Reidemeister torsion of $\mathcal{L}$ (see [Lipnowski 2014, §1.3]). It is a miraculous fact (see [Bismut and Zhang 1994, Theorem 0.2; Lipnowski 2014, Theorem 1.23]) that the twisted Reidemeister torsion is closely related to the twisted analytic torsion $\tau_{\sigma}(\mathcal{L})$, an equivariant spectral invariant of the metrized, equivariant local system $\mathcal{L}$ (described in [Lipnowski 2014, $\S 1.1])$. The main content of this paper is comparing $\tau_{\sigma}(\mathcal{L})$ to the untwisted analytic torsion $\tau(L)$ of a matching (see Section $4 \mathrm{C}$ ) local system. A prototypical example:

Sample Theorem (comparison of analytic torsion). Let $E / F$ be everywhere unramified. Let $\mathcal{U} \subset \boldsymbol{G}\left(\mathbb{A}_{E}^{\mathrm{fin}}\right)$ be a parahoric level structure (see Definition 5.1) and $U \subset \boldsymbol{G}\left(\mathbb{A}_{F}^{\text {fin }}\right)$ an associated level structure (see Definition 5.3). Then

$$
\tau_{\sigma}\left(\mathcal{M}_{\mathcal{U}}, \mathcal{L}\right)=\tau\left(M_{U}, L\right)^{p}
$$

(see Corollary 4.19 combined with Theorem 6.17 for a more general statement). By applying appropriate versions, both twisted and untwisted, of the Cheeger-Müller theorem (see [Lipnowski 2014, §§1-2]), we arrive at the equalities

$$
R_{\sigma}\left(\mathcal{M}_{\mathcal{U}}, \mathcal{L}\right) \sim \tau_{\sigma}\left(\mathcal{M}_{\mathcal{U}}, \mathcal{L}\right)=\tau\left(M_{U}, L\right)^{p}=R\left(M_{U}, L\right)^{p},
$$

where $\sim$ denotes equality up to powers of $p$. This reasoning is applicable to those matching pairs $\mathcal{L}, L$ of local systems described in Section $7 A$.

\section{Local systems over locally symmetric manifolds}

2A. Complex local systems over locally symmetric spaces. Let $\boldsymbol{H}$ be a semisimple group over a number field $F$. Let $K \subset H=\boldsymbol{H}\left(F_{\mathbb{R}}\right)$ be a maximal compact subgroup and $U \subset \boldsymbol{H}\left(\mathbb{A}_{F}^{\text {fin }}\right)$ a compact open subgroup. Assume $U$ is small enough that $\boldsymbol{H}(F)$ acts freely on $\boldsymbol{H}\left(\mathbb{A}_{F}\right) / K U$. Then the quotient $M_{U}=\boldsymbol{H}(F) \backslash \boldsymbol{H}\left(\mathbb{A}_{F}\right) / K U$ is a manifold. 
Let $W$ be a complex vector space and $\rho: H \rightarrow \mathrm{GL}(W)$ an irreducible representation. We form the local system

$$
L_{\rho}=\boldsymbol{H}(F) \backslash\left(\boldsymbol{H}\left(\mathbb{A}_{F}\right) / K U \times W\right) \rightarrow M_{U},
$$

where $\boldsymbol{H}(F)$ acts by $h \cdot\left(h^{\prime} K U, w\right)=\left(h h^{\prime} K U, \rho\left(h_{\infty}\right) w\right)$ and the bundle map is given by the first coordinate projection.

2A1. Equivariant local systems. Let $\Gamma$ be a finite group of automorphisms of $\boldsymbol{H}$. Suppose the action of $\Gamma$ preserves both $U$ and $K$. Then $\Gamma$ acts on the manifold $M_{U}$. Suppose further that the representation $\rho: H \rightarrow \mathrm{GL}(W)$ extends to a representation $\tilde{\rho}: H \rtimes \Gamma \rightarrow \mathrm{GL}(W)$. The action

$$
\Gamma \times L_{\rho} \rightarrow L_{\rho}, \quad(h K U, w) \mapsto(\sigma(h) K U, \tilde{\rho}(\sigma)(w))
$$

covers the action of $\Gamma$ on $M_{U}$. The action (4) gives $L_{\rho}$ the structure of an equivariant local system.

2A2. Riemannian structure. We refer to [Bergeron and Venkatesh 2013, §3.4] for a discussion of the normalizations used here.

Let $\theta$ be the Cartan involution corresponding to $K$ with associated decomposition $\mathfrak{h}:=\operatorname{Lie}\left(\boldsymbol{H}\left(F_{\mathbb{R}}\right)\right)=\mathfrak{k} \oplus \mathfrak{p}$ into the +1 -eigenspace $\mathfrak{k}=\operatorname{Lie}(K)$ and the -1 -eigenspace $\mathfrak{p}$.

By Weyl's unitary trick, the irreducible representation $\rho: \boldsymbol{H}\left(F_{\mathbb{R}}\right) \rightarrow \mathrm{GL}(W)$ corresponds to a unique representation, which we also call $\rho$, of the compact dual group $S$ of $H$; more precisely $S$ is the normalizer in $\boldsymbol{H}\left(F_{\mathbb{C}}\right)$ of the real Lie algebra $\mathfrak{k} \oplus i \mathfrak{p}$. There is a unique Hermitian metric on $W_{\iota}$ which is $S$-invariant, up to scaling. Fix such a choice of metric $\langle\cdot, \cdot\rangle_{0}$. We use this choice to define a metric $\|\cdot\|$ on the bundles $L_{\rho}$ :

$$
\|(h K U, v)\|^{2}=\left\|\rho\left(h_{\infty}^{-1}\right) v\right\|_{0}^{2} \quad \text { for }(h, v) \in L_{\rho} .
$$

Suppose a finite group $\Gamma$ acts on $\boldsymbol{H}$ by automorphisms and normalizes $K$ and $U$. Then $\Gamma$ also normalizes $S$ and so automatically preserves the metric $\|\cdot\|_{0}$. If the representation $\rho$ extends to a representation $\tilde{\rho}: H \rtimes \Gamma \rightarrow \operatorname{GL}(W)$, then $\Gamma$ acts on $L_{\rho}$, as in Section 2A1, by isometries.

2B. Rational and integral structures on local systems. Let $W$ be an $N$-vector space with $N / F$ a finite extension of number fields and let $\mathcal{O} \subset W$ be an $O_{N}$-lattice. Let $\rho: \boldsymbol{H} \rightarrow R_{N / F}$ GL(W) be an algebraic representation defined over $F$. Let $U \subset \boldsymbol{H}\left(\mathbb{A}_{F}^{\mathrm{fin}}\right)$ be a compact open subgroup and $K \subset \boldsymbol{H}\left(F_{\mathbb{R}}\right)$ a maximal compact subgroup with $X=\boldsymbol{H}\left(F_{\mathbb{R}}\right) / K$. Assume $U$ is small enough that $\boldsymbol{H}(F)$ acts freely on $\boldsymbol{H}\left(\mathbb{A}_{F}\right) / K U$. We form an associated local system of $N$-vector spaces

$$
L_{\rho}=\boldsymbol{H}(F) \backslash\left(\left(\boldsymbol{H}\left(\mathbb{A}_{F}\right) / K U\right) \times W\right) \rightarrow M_{U}=\boldsymbol{H}(F) \backslash \boldsymbol{H}\left(\mathbb{A}_{F}\right) / K U
$$


where $h \cdot(x, w)=(h x, \rho(h) w)$ for $h \in \boldsymbol{H}(F)$.

Now suppose that $\mathcal{O} \subset W$ is an $O_{N}$-lattice. The group $\boldsymbol{H}\left(\mathbb{A}_{F}^{\mathrm{fin}}\right)$ acts through $\rho$ on the space of $O_{N}$ lattices of $W$, and we suppose that $U$ stabilizes $\mathcal{O}$. Consider the local system of $O_{N}$-lattices over $X \times \boldsymbol{H}\left(\mathbb{A}_{F}^{\mathrm{fin}}\right) / U$ given by

$$
\Lambda_{\rho}^{0}:=\{(x, h U, v): v \in \rho(h) \mathcal{O}\} \rightarrow X \times \boldsymbol{H}\left(\mathbb{A}_{F}^{\mathrm{fin}}\right) / U
$$

with the bundle projection given by projection onto the first two factors. The group $\boldsymbol{H}(F)$ acts on $\Lambda_{\rho}^{0}$ diagonally: $h \cdot(x, g U, v)=\left(h_{\infty} x, h_{\text {fin }} g U, \rho(h) v\right)$. We let

$$
L_{\rho}^{0}:=\boldsymbol{H}(F) \backslash \Lambda_{\rho}^{0} \rightarrow M_{U}
$$

denote the quotient, which satisfies the following properties:

(1) $L_{\rho}^{0}$ is a local system of $O_{N}$-modules.

(2) $L_{\rho}^{0} \otimes_{O_{N}} N=L_{\rho}$.

(3) Let $\iota: N \hookrightarrow \mathbb{C}$ be a complex embedding. Let $\rho_{\iota}: \boldsymbol{H}\left(F_{\mathbb{R}}\right) \rightarrow \mathrm{GL}\left(W_{\iota}\right)$ be the composition

$$
\rho_{\iota}=\boldsymbol{H}\left(F_{\mathbb{R}}\right) \rightarrow \boldsymbol{H}\left(F_{\mathbb{C}}\right) \rightarrow \mathrm{GL}\left(W_{\iota}\right)
$$

Then

$$
\left(L_{\rho}^{0}\right) \otimes_{\iota} \mathbb{C}=L_{\rho_{\iota}} .
$$

Here, $L_{\rho_{l}}$ denotes the local system of $\mathbb{C}$-vector spaces associated to the complex representation $\rho_{l}$ in Section 2A.

Remark 2.1. Given an algebraic representation $\tilde{\rho}: R_{E / F} \boldsymbol{H} \rtimes \Gamma_{E / F} \rightarrow R_{N / F} \operatorname{GL}(W)$ defined over $F$, the constructions of this section apply equally well and give rise to $\mathcal{L}_{\tilde{\rho}}$ and $\mathcal{L}_{\tilde{\rho}}^{0}$, respectively equivariant local systems of $O_{N}$-modules and $N$-vector spaces satisfying

$$
\mathcal{L}_{\tilde{\rho}}^{0} \otimes_{O_{N}} N=\mathcal{L}_{\tilde{\rho}} \quad \text { and } \quad \mathcal{L}_{\tilde{\rho}} \otimes_{\iota} \mathbb{C}=\mathcal{L}_{\tilde{\rho}_{l}},
$$

where the latter is the equivariant local system of complex vector spaces constructed in Section 2A1.

\section{Zeta functions and analytic torsion of equivariant, metrized local systems}

3A. Equivariant analytic torsion over general manifolds. Let $\mathcal{M}$ be a compact Riemannian manifold and $\mathcal{L} \rightarrow \mathcal{M}$ a local system of $\mathbb{C}$-vector spaces equipped with a Hermitian metric. Let $\sigma$ act compatibly on $\mathcal{M}$ and $\mathcal{L}$ by a finite order isometry.

Remark 3.1. We do not require the parallel transport associated to the flat structure $\mathcal{L} \rightarrow \mathcal{M}$ to be unitary. 
Let $d_{\mathcal{L}}: \Omega^{\bullet}(\mathcal{M}, \mathcal{L}) \rightarrow \Omega^{\bullet}(\mathcal{M}, \mathcal{L})$ denote the exterior derivative, defined on local sections by $d_{\mathcal{L}}(\omega \otimes s)=d w \otimes s$. Let $d_{\mathcal{L}}^{*}$ denote the formal adjoint of $d_{\mathcal{L}}$. Then $\Delta=d_{\mathcal{L}} d_{\mathcal{L}}^{*}+d_{\mathcal{L}}^{*} d_{\mathcal{L}}$ is the Laplace operator associated to $\mathcal{L}$. We let $\Delta_{j}$ denote its restriction to $\Omega^{j}(\mathcal{M}, \mathcal{L})$.

Definition 3.2 (cf. [Lück 1993, (1.12)]). The $j$-th equivariant zeta function of $(\mathcal{L} \rightarrow \mathcal{M}, \sigma)$ is defined by

$$
\zeta_{j, \mathcal{L}, \sigma}(s)=\sum_{\lambda>0} \operatorname{tr}\left(\sigma \mid E_{\lambda}\right) \lambda^{-s}
$$

where the sum runs over the positive eigenvalues $\lambda$ of $\Delta_{j}$, with corresponding eigenspace $E_{\lambda}$.

The $j$-th zeta function of the metrized local system of complex vector spaces $L \rightarrow M$ over a compact Riemannian manifold is defined by

$$
\zeta_{j, L}:=\zeta_{j, L, \mathrm{id}}
$$

Each $\zeta_{j, \mathcal{L}, \sigma}$ admits a meromorphic continuation to the entire complex plane which is regular at $s=0$ [Lück 1993, Lemma 1.13].

Definition 3.3 [Lück 1993, Definition 1.14]. The equivariant analytic torsion of the triple $(\mathcal{L} \rightarrow \mathcal{M}, \sigma)$ is the quantity

$$
\tau_{\sigma}(\mathcal{L}):=\exp \left(-\frac{1}{2} \sum_{j=0}^{\operatorname{dim} M}(-1)^{j} j \cdot \zeta_{j, \mathcal{L}, \sigma}^{\prime}(0)\right) .
$$

The untwisted analytic torsion (or simply analytic torsion) of a metrized local system $L \rightarrow M$ over a compact Riemannian manifold $M$ is defined to be $\tau(L):=\tau_{\text {id }}(L)$.

3B. Equivariant analytic torsion over locally symmetric manifolds. Let $\boldsymbol{H}$ be a semisimple algebraic group over a number field $F$. Let $E / F$ be a cyclic Galois extension of degree $p$ with Galois group $\Gamma_{E / F}=\langle\sigma\rangle$.

Let $\mathcal{K} \subset \widetilde{H}:=\boldsymbol{H}\left(E_{\mathbb{R}}\right)$ be maximal compact with corresponding Cartan decomposition $\tilde{\mathfrak{h}}=\tilde{\mathfrak{k}} \oplus \tilde{\mathfrak{p}}$.

If the Casimir operator of a group $\bullet$ acts on a representation $r$ of $\bullet$ by a scalar, let $\lambda_{r}$ denote this scalar.

Let $\mathcal{U} \subset \boldsymbol{H}\left(\mathbb{A}_{E}^{\text {fin }}\right)$ be compact open. Assume that both $\mathcal{K}$ and $\mathcal{U}$ are Galois-stable. Assume that $\mathcal{U}$ is small enough that the quotient $\mathcal{M}_{\mathcal{U}}:=\boldsymbol{H}(E) \backslash \boldsymbol{H}\left(\mathbb{A}_{E}\right) / \mathcal{K U}$ is a manifold. Assume that $\boldsymbol{H}$ is anisotropic over $E$; this is equivalent to $\mathcal{M}_{\mathcal{U}}$ being compact.

Let $\tilde{\rho}: \widetilde{H} \rtimes \Gamma_{E / F} \rightarrow \mathrm{GL}(W)$ be a complex representation. Let $\mathcal{L}_{\tilde{\rho}} \rightarrow \mathcal{M}_{\mathcal{U}}$ be the $\Gamma_{E / F}$-equivariant local system associated to $\tilde{\rho}$ (see Section $2 \mathrm{~A} 1$ ) endowed with the Hermitian structure described in Section 2A2. 
Denote the $\boldsymbol{H}\left(\mathbb{A}_{E}\right) \rtimes \Gamma_{E / F}$-isotypic decomposition of $L^{2}\left(\boldsymbol{H}(E) \backslash \boldsymbol{H}\left(\mathbb{A}_{E}\right)\right)$ by

$$
L^{2}\left(\boldsymbol{H}(E) \backslash \boldsymbol{H}\left(\mathbb{A}_{E}\right)\right)=\bigoplus_{\tilde{\pi}} \mathcal{W}[\tilde{\pi}] .
$$

The equivariant zeta functions $\zeta_{j, L, \sigma}$ can be expressed purely in terms of the $\tilde{\pi}$. Before stating a precise result, we set some representation theory notation.

Notation for trace. For a $\sigma$-module $A$, we let $\langle A\rangle$ denote $\operatorname{tr}\{\sigma \mid A\}$.

Definition 3.4. An admissible, irreducible representation $\tilde{\pi}$ of $\boldsymbol{H}\left(\mathbb{A}_{E}\right) \rtimes \Gamma_{E / F}$ is called essential if $\left.\tilde{\pi}\right|_{\boldsymbol{H}\left(\mathbb{A}_{E}\right)}$ is irreducible. It is called inessential otherwise.

Suppose that $\tilde{\pi}=\tilde{\pi}_{\infty} \otimes \tilde{\pi}_{\text {fin }}$ is a representation of $\boldsymbol{H}\left(\mathbb{A}_{E}\right)$. Suppose further that the action of $\sigma$ on $\tilde{\pi}$ factorizes as $\tilde{\pi}(\sigma)=\tilde{\pi}_{\infty}\left(\sigma_{\infty}\right) \otimes \tilde{\pi}_{\text {fin }}\left(\sigma_{\text {fin }}\right)$.

Remark 3.5. Let $\boldsymbol{H}$ be the adjoint group of $\underline{D}^{\times}$for a quaternion algebra over $F$. For every essential representation $\tilde{\pi}$ of $\boldsymbol{H}\left(\mathbb{A}_{E}\right) \rtimes\langle\sigma\rangle$, we have that $\tilde{\pi}(\sigma)$ admits a preferred factorization $\tilde{\pi}(\sigma)=\otimes_{v}^{\prime} \tilde{\pi}_{v}\left(\sigma_{v}\right)$. See our discussion of base change, especially Theorem 4.11 .

Lemma 3.6. The $j$-th equivariant zeta function of $\left(\mathcal{L}_{\tilde{\rho}} \rightarrow \mathcal{M}_{\mathcal{U}}, \sigma\right)$ equals

$$
\zeta_{j, \mathcal{M}_{\mathcal{U}}, \mathcal{L}_{\tilde{\rho}}, \sigma}(s)=\sum_{\lambda} \lambda^{-s} \sum_{\substack{\tilde{\pi} \\ \lambda_{\tilde{\rho}}-\lambda_{\tilde{\pi}_{\infty}}=\lambda}} m(\tilde{\pi}) \cdot\left\langle\tilde{\pi}_{\text {fin }}^{\mathcal{U}}\right\rangle \cdot\left\langle\operatorname{Hom}_{\mathcal{K}}\left(\wedge^{j} \tilde{\mathfrak{p}}, \tilde{\pi}_{\infty} \otimes \tilde{\rho}\right)\right\rangle,
$$

where $m(\tilde{\pi}):=\operatorname{dim} \operatorname{Hom}_{\boldsymbol{H}\left(\mathbb{A}_{E}\right) \rtimes \Gamma_{E / F}}(\tilde{\pi}, \mathcal{W})$.

Proof. See [Fung 2002, §2.3; Speh 1994]. The key inputs are as follows:

- The $\mathcal{L}_{\tilde{\rho}}$-valued differential forms on $\mathcal{M}_{\mathcal{U}}$ decompose as a (Hilbert) direct sum:

$$
\Omega^{j}\left(\mathcal{M}_{\mathcal{U}}\right)=\bigoplus_{\tilde{\pi}} \operatorname{Hom}_{\mathcal{K}}\left(\wedge^{j} \tilde{\mathfrak{p}}, \mathcal{W}[\tilde{\pi}]^{\mathcal{U}} \otimes \tilde{\rho}\right)
$$

Kuga's lemma [Borel and Wallach 2000, Chapter II, §2] states that $\Delta_{\mathcal{L}_{\tilde{\rho}}}$ acts on $\operatorname{Hom}_{\mathcal{K}}\left(\wedge^{j} \tilde{\mathfrak{p}}, \mathcal{W}[\tilde{\pi}]^{\mathcal{U}} \otimes \tilde{\rho}\right)$ by the scalar $\lambda_{\tilde{\rho}}-\lambda_{\tilde{\pi}}$.

- If $\tilde{\pi}$ is inessential, then $\left.\tilde{\pi}\right|_{\boldsymbol{H}\left(\mathbb{A}_{E}\right)}$ decomposes as a direct sum of $p$ (irreducible) representations $V_{1} \oplus \cdots \oplus V_{p}$ where $\tilde{\pi}(\sigma)$ cyclically permutes the $V_{i}$. In particular, because no summand $V_{i}$ is preserved,

$$
\left\langle\operatorname{Hom}_{\mathcal{K}}\left(\wedge^{j} \tilde{\mathfrak{p}}, \mathcal{W}[\tilde{\pi}]^{\mathcal{U}} \otimes \tilde{\rho}\right)\right\rangle=0 \quad \text { for inessential } \tilde{\pi} .
$$

Corollary 3.7. The $j$-th zeta function of the metrized local system $L_{\rho} \rightarrow M_{U}$ equals

$$
\zeta_{j, M_{U}, L_{\rho}}(s)=\sum_{\lambda} \lambda^{-s} \sum_{\substack{\pi \\ \lambda_{\rho_{l}}-\lambda_{\pi_{\infty}}=\lambda}} m(\pi) \cdot \operatorname{dim} \pi_{\mathrm{fin}}^{U} \cdot \operatorname{dim}\left(\operatorname{Hom}_{K}\left(\wedge^{j} \mathfrak{p}, \pi_{\infty} \otimes \rho\right)\right) .
$$


Proof. Lemma 3.6 specializes to the corollary statement when $E=F, \sigma=\mathrm{id}$, $\mathcal{U}=U, \mathcal{K}=K, \tilde{\rho}=\rho$.

\section{Base change for quaternion algebras and analytic torsion}

Let $\boldsymbol{G}$ be the adjoint group of a quaternion algebra $D$ over a number field $F$. Let $E / F$ be a cyclic Galois extension of odd prime degree $p$ with Galois group $\Gamma_{E / F}=\langle\sigma\rangle$. Assume that $D_{E}$ is not split. Then $\boldsymbol{G}(E) \backslash \boldsymbol{G}\left(\mathbb{A}_{E}\right)$ and $\boldsymbol{G}(F) \backslash \boldsymbol{G}\left(\mathbb{A}_{F}\right)$ are compact.

Let $\mathcal{M}_{\mathcal{U}}=\boldsymbol{G}(E) \backslash \boldsymbol{G}\left(\mathbb{A}_{E}\right) / \mathcal{K U}$ for some Galois-stable maximal compact subgroup $\mathcal{K} \subset \boldsymbol{G}\left(E_{\mathbb{R}}\right)$ and some Galois-stable compact open subgroup $\mathcal{U} \subset \boldsymbol{G}\left(\mathbb{A}_{E}^{\mathrm{fin}}\right)$. Let $M_{U}=\boldsymbol{G}(F) \backslash \boldsymbol{G}\left(\mathbb{A}_{F}\right) / K U$ for some maximal compact $K \subset \boldsymbol{G}\left(F_{\mathbb{R}}\right)$ and some compact open $U \subset \boldsymbol{G}\left(\mathbb{A}_{F}^{\mathrm{fin}}\right)$.

One main goal of this paper is to prove a spectral comparison of the flavor

$$
\log \tau_{\sigma}\left(\mathcal{M}_{\mathcal{U}}, \mathcal{L}\right)=p \log \tau\left(M_{U}, L\right)
$$

for appropriate pairs of matching local systems of complex vector spaces $\mathcal{L} \rightarrow \mathcal{M}_{\mathcal{U}}$ and $L \rightarrow M_{U}$.

We will prove such spectral identities using trace formula techniques, by comparing the trace of a twisted convolution operator on $L^{2}\left(\boldsymbol{G}(E) \backslash \boldsymbol{G}\left(\mathbb{A}_{E}\right)\right)$ to that of an untwisted convolution operator on $L^{2}\left(\boldsymbol{G}(F) \backslash \boldsymbol{G}\left(\mathbb{A}_{F}\right)\right)$, exactly in the spirit of Langlands' book [1980] on base change for $\mathrm{GL}_{2}$.

In Section 4A, we will discuss those elements of the theory of base change needed to prove the comparison (8). In Section $4 \mathrm{~B}$, we discuss local-global compatibility of base change and a consequence for "multiplicities". In Section 4C, we discuss matching representations and matching local systems. Such matching pairs occur in the abstract matching theorem proved in Section 4D. Later, in Sections 5 and 6, instances of test functions satisfying the hypotheses of the abstract matching theorem will be described.

4A. Preliminaries on base change. For general references on base change, see [Langlands 1980] for $\mathrm{GL}_{2}$ and [Arthur and Clozel 1989] for $\mathrm{GL}_{n}$. For a comprehensive treatment of the twisted trace formula formalism, see [Labesse and Waldspurger 2013].

4A1. Twisted convolution and convolution.

Definition 4.1. Let $\operatorname{SM}_{c}\left(\boldsymbol{G}\left(\mathbb{A}_{E}\right)\right)$ denote the space of smooth compactly supported measures on $\boldsymbol{G}\left(\mathbb{A}_{E}\right)$. More precisely, these are all finite linear combinations of measures of the form $\tilde{f} d \tilde{g}$ for a Haar measure $d \tilde{g}$ on $\boldsymbol{G}\left(\mathbb{A}_{E}\right)$ and a function $f=\prod_{v} f_{v}$. We require that $f_{\infty}$ be smooth and compactly supported, that $f_{v}$ be compactly supported and locally constant, and that $f_{v}=\mathbf{1}_{G\left(O_{E_{v}}\right)}$ for almost all places $v$ of $F$. We define $\operatorname{SM}_{c}\left(\boldsymbol{G}\left(\mathbb{A}_{F}\right)\right)$ similarly. 
For any smooth, compactly supported measure $\tilde{f} d \tilde{g}$ on $\boldsymbol{G}\left(\mathbb{A}_{E}\right)$, let

$$
\mathcal{R}(\tilde{f} d \tilde{g})=\int_{\boldsymbol{G}\left(\mathbb{A}_{E}\right)} \tilde{f}(g) \mathcal{R}(g) d \tilde{g} \circlearrowleft L^{2}\left(\boldsymbol{G}(E) \backslash \boldsymbol{G}\left(\mathbb{A}_{E}\right)\right),
$$

where $\mathcal{R}$ denotes the right regular representation of $\boldsymbol{G}\left(\mathbb{A}_{E}\right)$ acting on $\boldsymbol{G}(E) \backslash \boldsymbol{G}\left(\mathbb{A}_{E}\right)$. Note that the larger group $\boldsymbol{G}\left(\mathbb{A}_{E}\right) \rtimes \Gamma_{E / F}$ acts on $\boldsymbol{G}(E) \backslash \boldsymbol{G}\left(\mathbb{A}_{E}\right)$.

Similarly, for a smooth, compactly supported measure $f d g$ on $\boldsymbol{G}\left(\mathbb{A}_{F}\right)$, we let

$$
r(f d g)=\int_{\boldsymbol{G}\left(\mathbb{A}_{F}\right)} f(g) r(g) d g \circlearrowleft L^{2}\left(\boldsymbol{G}(F) \backslash \boldsymbol{G}\left(\mathbb{A}_{F}\right)\right),
$$

where $r$ denotes the right regular representation of $\boldsymbol{G}\left(\mathbb{A}_{F}\right)$.

4A2. Twisted conjugacy and the norm map. Suppose that $E_{v}=E \otimes F_{v}$ is unramified over $F_{v}$, i.e., is either a split extension or an unramified field extension. We define the norm map [Langlands 1980, §4]

$$
N: \boldsymbol{G}\left(E_{v}\right) \rightarrow \boldsymbol{G}\left(E_{v}\right), \quad x \mapsto x \sigma(x) \cdots \sigma^{p-1}(x) .
$$

Lemma 4.2 (cf. [Arthur and Clozel 1989, Lemma 1.1(i)]). If $x \in \boldsymbol{G}\left(E_{v}\right)$, then $N x$ is $\boldsymbol{G}\left(E_{v}\right)$-conjugate to an element of $y$ of $\boldsymbol{G}\left(F_{v}\right)$, where $y$ is uniquely defined modulo $\boldsymbol{G}\left(F_{v}\right)$-conjugation.

Proof. Lift $x$ to $\tilde{x}$ in $D_{E_{v}}^{\times}$. We can define a norm map on $D_{E_{v}}^{\times}$using formula (9); we also denote it by $N$. If $\tilde{u}=N(\tilde{x})$, then

$$
\sigma(\tilde{u})=\tilde{x}^{-1} \tilde{u} \tilde{x} .
$$

The characteristic polynomial $p_{\tilde{u}}(t)=t^{2}-\operatorname{trd}(\tilde{u}) t+\operatorname{nrd}(\tilde{u}) \in E_{v}[t]$ is thus preserved by $\Gamma_{E / F}$. Therefore, $p_{\tilde{u}}(t) \in F_{v}[t]$.

If $\tilde{u} \in F_{v}^{\times} \subset D_{F_{v}}^{\times}$, we are done. Otherwise, the polynomial $p_{\tilde{u}}$ is irreducible. Since the quaternion algebra $D_{F_{v}}$ contains all quadratic extensions of $F_{v}$, there is some element $\tilde{u}^{\prime} \in D_{F_{v}}$ with the same characteristic polynomial as $N \tilde{x} \in D_{E_{v}}$. By the Noether-Skolem theorem for $D_{E_{v}}$, we know that $N \tilde{x}$ and $\tilde{u}^{\prime}$ are conjugate in $D_{E_{v}}^{\times}$, implying that the image of $\tilde{u}^{\prime}$ in $\boldsymbol{G}\left(F_{v}\right)$ and $N x$ are conjugate by $\boldsymbol{G}\left(E_{v}\right)$. By a second application of the Noether-Skolem theorem, any two elements of $\boldsymbol{G}\left(F_{v}\right)$ which are $\boldsymbol{G}\left(E_{v}\right)$-conjugate are $\boldsymbol{G}\left(F_{v}\right)$-conjugate.

Lemma 4.3 (cf. [Arthur and Clozel 1989, Lemma 1.1(ii)]). If $N x$ and $N y$ are $\boldsymbol{G}\left(E_{v}\right)$-conjugate, then $x$ and $y$ are $\sigma$-conjugate.

Proof. Applying $\sigma$-conjugation to $x$ and $y$ as necessary, we may suppose that $N x=N y \in \boldsymbol{G}\left(F_{v}\right)$. Lift $x, y$ to $\tilde{x}, \tilde{y} \in D_{E_{v}}^{\times}$. Then

$$
N \tilde{x}=c N \tilde{y}
$$


for some $c \in E_{v}^{\times}$. This implies that

$$
c^{2}=\operatorname{Norm}_{E_{v} / F_{v}}\left(\operatorname{nrd}\left(\tilde{x} \tilde{y}^{-1}\right)\right) \in \operatorname{Norm}_{E_{v} / F_{v}}\left(E_{v}^{\times}\right) \subset F_{v}^{\times} .
$$

Since $\left[E_{v}: F_{v}\right]=p$ is odd, we have $c \in F_{v}^{\times}$. Furthermore, $F_{v}^{\times} / \operatorname{Norm}_{E_{v} / F_{v}}\left(E_{v}^{\times}\right)$is isomorphic to $\operatorname{Gal}\left(E_{v} / F_{v}\right)$ by class field theory. Since $\operatorname{Gal}\left(E_{v} / F_{v}\right)$ has odd order $p$, it follows that $c=\operatorname{Norm}_{E_{v} / F_{v}}\left(c^{\prime}\right)$ for some $c^{\prime} \in E_{v}^{\times}$. Replace $\tilde{y}$ by $\tilde{y}^{\prime}=c^{\prime} \tilde{y}$. By Noether-Skolem, $N \tilde{y}^{\prime}$ and $N \tilde{x}$ are $D_{F_{v}}^{\times}$-conjugate. The argument from [Langlands 1980, Lemma 4.2] then shows that $\tilde{x}$ and $\tilde{y}^{\prime}$ are $\sigma$-conjugate.

Corollary 4.4. The norm map induces a well-defined injection

$N:\left\{\sigma\right.$-twisted conjugacy classes in $\left.\boldsymbol{G}\left(E_{v}\right)\right\} \rightarrow$ conjugacy classes in $\left.\boldsymbol{G}\left(F_{v}\right)\right\}$,

$$
[x] \mapsto[N(x)] \cap \boldsymbol{G}\left(F_{v}\right) .
$$

Proof. The norm map converts $\sigma$-twisted conjugacy to conjugacy because of the fact that $N\left(g^{-1} x \sigma(g)\right)=g^{-1} N(x) g$. The rest follows from Lemmas 4.2 and 4.3.

Corollary 4.5. If $x, y \in \boldsymbol{G}\left(F_{v}\right)$ are $\boldsymbol{G}\left(\bar{F}_{v}\right)$-conjugate, then they are $\boldsymbol{G}\left(F_{v}\right)$-conjugate. If $x^{\prime}, y^{\prime} \in \boldsymbol{G}\left(E_{v}\right)$ are $\boldsymbol{G}\left(\bar{E}_{v}\right) \sigma$-conjugate, then they are $\boldsymbol{G}\left(E_{v}\right) \sigma$-conjugate.

Proof. The first part follows by the same result for $\underline{D}_{F_{v}}^{\times}$; this in turn follows by the Noether-Skolem theorem.

For the second part, the assumptions imply that $N x^{\prime}, N y^{\prime} \in \boldsymbol{G}\left(F_{v}\right)$ are $\boldsymbol{G}\left(\bar{F}_{v}\right)$ conjugate. By the first part, it follows that $N x^{\prime}$ and $N y^{\prime}$ are $\boldsymbol{G}\left(F_{v}\right)$-conjugate. By Lemma 4.3, it follows that $x^{\prime}, y^{\prime}$ are $\boldsymbol{G}\left(E_{v}\right) \sigma$-conjugate.

4A3. Matching orbital integrals. See [Langlands 1980, §6] for a discussion of orbital integrals in the context of base change for $\mathrm{GL}_{2}$.

For a $\sigma$-twisted conjugacy class $c=[x]$ of $\boldsymbol{G}\left(E_{v}\right)$, a conjugacy class $c^{\prime}=\left[x^{\prime}\right]$ of $\boldsymbol{G}\left(F_{v}\right)$, and test measures $f d g$ on $\boldsymbol{G}\left(F_{v}\right)$ and $\tilde{f} d \tilde{g}$ on $\boldsymbol{G}\left(E_{v}\right)$, we let

$$
\begin{aligned}
\mathcal{O}_{\sigma, c}(\tilde{f} d \tilde{g}) & =\int_{\boldsymbol{G}_{\sigma \ltimes x}\left(F_{v}\right) \backslash \boldsymbol{G}\left(E_{v}\right)} \tilde{f}\left(g^{-1} x \sigma(g)\right) \frac{d \tilde{g}}{d z_{\sigma}}, \\
\mathcal{O}_{c^{\prime}}(f d g) & =\int_{\boldsymbol{G}_{x^{\prime}}\left(F_{v}\right) \backslash \boldsymbol{G}\left(F_{v}\right)}^{f\left(g^{-1} x^{\prime} g\right) \frac{d g}{d z},}
\end{aligned}
$$

where $\boldsymbol{G}_{\sigma \ltimes x}$ denotes the twisted centralizer of $x$ and $\boldsymbol{G}_{x^{\prime}}$ denotes the centralizer of $x^{\prime}$. See Remark 4.7 for a discussion of the Haar measures $d z$ and $d z_{\sigma}$.

Definition 4.6. We say that $\tilde{f} d \tilde{g}$ and $f d g$ match if $\mathcal{O}_{c^{\prime}}(f d g)= \begin{cases}\mathcal{O}_{\sigma, c}(\tilde{f} d \tilde{g}) & \text { if } c \text { is a regular twisted conjugacy class and } c^{\prime}=N c, \\ 0 & \text { if } c^{\prime} \neq N c \text { for any twisted conjugacy class } c .\end{cases}$

Similarly, we say that the pure tensors $\tilde{f} d \tilde{g}=\prod_{v} \tilde{f}_{v} d \tilde{g}_{v} \in \operatorname{SM}_{c}\left(\boldsymbol{G}\left(\mathbb{A}_{E}\right)\right)$ and $f d g=\prod_{v} f_{v} d g_{v} \in \operatorname{SM}_{c}\left(\boldsymbol{G}\left(\mathbb{A}_{F}\right)\right)$ match if they match everywhere locally. 
Remark 4.7. The definitions of $\mathcal{O}_{\sigma, \delta}$ and $\mathcal{O}_{\gamma}$ depend on choices of Haar measures $d z_{\sigma}$ on $\boldsymbol{G}_{\sigma \ltimes \delta}\left(F_{v}\right)$ and $d z$ on $\boldsymbol{G}_{\gamma}\left(F_{v}\right)$. However, if $\gamma=N \delta$, then the $F$-algebraic groups $\boldsymbol{G}_{\gamma}$ and $\boldsymbol{G}_{\sigma \ltimes \delta}$ are inner forms. Therefore, a choice of Haar measure $d z_{\sigma}$ on $\boldsymbol{G}_{\sigma \ltimes \delta}\left(F_{v}\right)$ determines a compatible Haar measure $d z$ on $\boldsymbol{G}_{\gamma}\left(F_{v}\right)$ (see [Kottwitz 1982, Lemma 5.8]). The equality from Definition 4.6 is to be taken with respect to this compatible choice. Though the individual orbital integrals depend on choices of Haar measure, this convention ensures that the notion of $\tilde{f} d \tilde{g}$ and $f d g$ matching is independent of all choices.

Example 4.8 [Langlands 1980, §8]. Say that $E_{v} / F_{v}$ is split, i.e., $E_{v}=F_{v}^{p}$. Then for any test function $f_{1} d g_{1} \times \cdots \times f_{p} d g_{p}$ on $\boldsymbol{G}\left(E_{v}\right)=\boldsymbol{G}\left(F_{v}\right)^{p}$, the convolution product $\left(f_{1} d g_{1}\right) * \cdots *\left(f_{p} d g_{p}\right)$ on $\boldsymbol{G}\left(F_{v}\right)$ matches.

4A4. Statement of base change. For this section, let $W=L^{2}\left(\boldsymbol{G}(F) \backslash \boldsymbol{G}\left(\mathbb{A}_{F}\right)\right)$ and $\mathcal{W}=L^{2}\left(\boldsymbol{G}(E) \backslash \boldsymbol{G}\left(\mathbb{A}_{E}\right)\right)$.

Theorem 4.9 (Saito, Shintani, Langlands). Let $\tilde{f} d \tilde{g}=\prod_{v} \tilde{f}_{v} d \tilde{g}_{v} \in \operatorname{SM}_{c}\left(\boldsymbol{G}\left(\mathbb{A}_{E}\right)\right)$ and $f d g=\prod_{v} f_{v} d g_{v} \in \operatorname{SM}_{c}\left(\boldsymbol{G}\left(\mathbb{A}_{F}\right)\right)$ be matching test functions. Then

$$
\operatorname{tr}\{\mathcal{R}(\sigma) \mathcal{R}(\tilde{f} d \tilde{g}) \mid \mathcal{W}\}=\operatorname{tr}\{r(f d g) \mid W\} .
$$

Of course, the onus is on us to produce interesting examples of matching test functions. The identity (10) can be leveraged to give a more refined identity.

Corollary 4.10 [Gelbart and Jacquet 1979]. Let E/F be a cyclic Galois extension of number fields of odd prime degree. There is a bijection between irreducible automorphic representations $\pi$ of $\boldsymbol{G}$ and essential (see Definition 3.4) automorphic representations $\tilde{\pi}$ of $R_{E / F} \boldsymbol{G} \rtimes \Gamma_{E / F}$. This bijection is uniquely defined by an equality of traces: $\pi$ and $\tilde{\pi}$ match if and only if

$$
\operatorname{tr}\{\mathcal{R}(\sigma) \mathcal{R}(\tilde{f} d \tilde{g}) \mid \mathcal{W}[\tilde{\pi}]\}=\operatorname{tr}\{r(f d g) \mid W[\pi]\}
$$

for all pairs of matching test functions $\tilde{f} d \tilde{g}$ and $f d g$ as above. Here, $\mathcal{W}[\tilde{\pi}]$ denotes the $\tilde{\pi}$ isotypic subspace of $\mathcal{W}$ and $W[\pi]$ the $\pi$-isotypic subspace of $W$.

\section{B. Local-global compatibility for base change and a consequence for multiplic-} ities. The groups $R_{E / F} \boldsymbol{G}_{E}$ and $\boldsymbol{G}$ satisfy multiplicity one, by the Jacquet-Langlands correspondence and multiplicity one for $\mathrm{GL}_{2}$. Therefore, the equality of (11) is equivalent to

$$
\operatorname{tr}\{\tilde{\pi}(\sigma) \tilde{\pi}(\tilde{f} d \tilde{g})\}=\operatorname{tr}\{\pi(f d g)\}
$$

for all pairs of matching test functions $\tilde{f} d \tilde{g}$ and $f d g$. Using (12), Langlands proves a local version of base change and local-global compatibility.

Theorem 4.11 [Langlands 1980, §7]. Let E/F be a cyclic Galois extension of odd prime degree $p$. There is a bijection between irreducible representations $\pi_{v}$ 
of $\boldsymbol{G}\left(F_{v}\right)$ and representations $\tilde{\pi}_{v}$ of $\boldsymbol{G}\left(E_{v}\right) \rtimes \Gamma_{E / F}$ which are irreducible after restricting to $\boldsymbol{G}\left(E_{v}\right)$. This bijection is determined by the equality of traces

$$
\operatorname{tr}\left\{\tilde{\pi}_{v}\left(\sigma_{v}\right) \tilde{\pi}_{v}\left(\tilde{f}_{v} \tilde{g}_{v}\right)\right\}=\operatorname{tr}\left\{\pi_{v}\left(f_{v} d g_{v}\right)\right\}
$$

for all pairs of matching test functions $\tilde{f}_{v} d \tilde{g}_{v}$ and $f_{v} d g_{v}$. The image of $\tilde{\pi}_{v}$ under this bijection is often denoted $\mathrm{BC}\left(\pi_{v}\right)$ and referred to as the base change of $\pi_{v}$. This base change bijection is compatible with the global bijection of Corollary 4.10 in the sense that $\pi=\otimes^{\prime} \pi_{v}$ globally matches the unique essential representation $\tilde{\pi}=\otimes^{\prime} \tilde{\pi}_{v}$.

Remark 4.12. As promised in Remark 3.5, the above local-global compatibility theorem has the following consequence: for any essential representation $\tilde{\pi}=\otimes^{\prime} \tilde{\pi}_{v}$ of $\boldsymbol{G}\left(\mathbb{A}_{E}\right) \rtimes \Gamma_{E / F}$ matching the representation $\pi=\otimes^{\prime} \pi_{v}$ of $\boldsymbol{G}\left(\mathbb{A}_{F}\right)$, there is a canonical factorization

$$
\tilde{\pi}(\sigma)=\otimes^{\prime} \tilde{\pi}\left(\sigma_{v}\right)
$$

4B1. Local base change and "trace matching".

Definition 4.13. Let $\tilde{f} d \tilde{g}$ be a smooth measure on $\boldsymbol{G}\left(E_{v}\right)$ and let $f d g$ be a smooth measure on $\boldsymbol{G}\left(F_{v}\right)$. We say that $\tilde{f} d \tilde{g}$ and $f d g$ are trace-matching if, for every irreducible admissible representation $\pi$ of $\boldsymbol{G}\left(F_{v}\right)$ with base change representation $\mathrm{BC}(\pi)$ of $\boldsymbol{G}\left(E_{v}\right)$, there is an equality

$$
\operatorname{tr}\{\mathrm{BC}(\pi)(\sigma) \mathrm{BC}(\pi)(\tilde{f} d \tilde{g})\}=\operatorname{tr}\{\pi(f d g)\} .
$$

Example 4.14. If $\tilde{f} d \tilde{g}$ and $f d g$ have matching orbital integrals in the sense of Definition 4.6, then they are trace-matching.

We record one straightforward consequence that Theorem 4.11 on local-global compatibility of base change has for comparing "multiplicities":

Lemma 4.15. Let $S$ be any set of places. Let $\mathcal{U}=\prod_{v \in S} \mathcal{U}_{v}$ be a compact open subgroup of the restricted tensor product $\prod_{v \in S}^{\prime} \boldsymbol{G}\left(E_{v}\right)$. Suppose that $\mathbf{1}_{\mathcal{U}_{v}} d u_{v}$ tracematches $m_{v}$ for each $v \in S$ and that $\prod_{v \in S} m_{v}=\sum c_{U} \mathbf{1}_{U} d u$. Then for matching representations $\tilde{\pi}=\otimes_{v}^{\prime} \tilde{\pi}_{v}$ of $\boldsymbol{G}\left(\mathbb{A}_{E}\right) \rtimes \Gamma_{E / F}$ and $\pi=\otimes \pi_{v}$ of $\boldsymbol{G}\left(\mathbb{A}_{F}\right)$, there is an equality

$$
\left\langle\tilde{\pi}_{S}^{\mathcal{U}}\right\rangle=\sum_{U} c_{U} \operatorname{dim} \pi_{S}^{U}
$$

where $\bullet_{S}$ denotes the (restricted) tensor or cartesian product over all places $v \in S$. 
Proof. This follows directly by Theorem 4.11 and Section 4B1 of trace-matching:

$$
\begin{aligned}
\left\langle\tilde{\pi}_{S}^{\mathcal{U}}\right\rangle & :=\operatorname{tr}\left\{\tilde{\pi}_{S}(\sigma) \tilde{\pi}_{S}\left(\mathbf{1}_{\mathcal{U}} d u\right)\right\}=\prod_{v \in S} \operatorname{tr}\left\{\tilde{\pi}_{v}(\sigma) \tilde{\pi}_{v}\left(\mathbf{1}_{\mathcal{U}_{v}} d \tilde{u}_{v}\right)\right\} \\
& =\prod_{v \in S} \operatorname{tr}\left\{\pi\left(m_{v}\right)\right\}=\operatorname{tr}\left\{\pi_{S}\left(\sum c_{U} \mathbf{1}_{U} d u\right)\right\}=\sum_{U} c_{U} \operatorname{dim} \pi_{S}^{U} .
\end{aligned}
$$

4C. Local systems and matching representations. Let $\boldsymbol{H}$ be a semisimple group over the number field $F$. Let $i_{E}: F \hookrightarrow E$ be a cyclic Galois extension of prime degree $p$. Note that $\left(R_{E / F} \boldsymbol{H}\right)_{E}=\prod_{\Gamma_{E / F}} \boldsymbol{H}_{E}$, where $\Gamma_{E / F}$ acts by permuting the factors according to its left translation action on itself. Let $i_{N}: F \hookrightarrow N$ be a second finite extension. Let $R_{E / F} \boldsymbol{H} \rightarrow R_{N / F} \mathrm{GL}(V)$, for $V$ a finite dimensional $N$-vector space, be an algebraic homomorphism defined over $F$.

4C1. Definition and examples of matching representations and local systems. Suppose $\iota: N \hookrightarrow \mathbb{C}$ is a complex embedding and suppose $\mathbb{C}_{\iota}=N \otimes_{\iota} \mathbb{C}$. Then $R_{E / F} \boldsymbol{H}\left(\mathbb{C}_{\iota}\right)=\prod_{\iota^{\prime}} \boldsymbol{H}\left(\mathbb{C}_{\iota^{\prime}}\right)$ where the product runs over all $\iota^{\prime}: E \hookrightarrow \mathbb{C}$ extending $\iota \circ i_{N}$. The Galois group $\Gamma_{E / F}$ acts on this product by permuting the factors according to its action on the set $\left\{\iota^{\prime} \mid \iota \circ i_{N}\right\}$. Furthermore, the induced homomorphism $R_{E / F} \boldsymbol{H}(F \otimes \mathbb{R}) \rightarrow \mathrm{GL}(V)\left(\mathbb{C}_{l}\right)$ factors as

$$
R_{E / F} \boldsymbol{H}(F \otimes \mathbb{R}) \rightarrow R_{E / F} \boldsymbol{H}(F \otimes \mathbb{C}) \rightarrow R_{E / F} \boldsymbol{H}\left(\mathbb{C}_{l}\right) \rightarrow \mathrm{GL}(V)\left(\mathbb{C}_{l}\right),
$$

the second map being induced by the projection $F \otimes \mathbb{C} \rightarrow \mathbb{C}_{\iota}$.

Definition 4.16 (matching representations for different coefficient fields). Suppose $\tilde{\rho}: R_{E / F} \boldsymbol{H} \rtimes \Gamma_{E / F} \rightarrow R_{N / F} \mathrm{GL}\left(V_{\tilde{\rho}}\right)$ and $\rho: \boldsymbol{H} \rightarrow R_{N / F} \mathrm{GL}\left(V_{\rho}\right)$ are representations of algebraic groups over $F$. Let $\iota: N \hookrightarrow \mathbb{C}$ be a complex embedding. We say that $\tilde{\rho}$ l-matches $\rho$ if

$$
\tilde{\rho}_{\iota}=\otimes_{\iota^{\prime} \mid \iota} \rho_{\iota},
$$

with $\Gamma_{E / F}$ acting by permutations on the set $\left\{\iota^{\prime} \mid \iota \circ i_{N}\right\}$. When no confusion will result, we abbreviate "l-match" to "match". For local systems $V_{\tilde{\rho}}$ and $V_{\rho}$ of $N$-vector spaces arising in the manner of Section $2 \mathrm{~B}$, we say that $V_{\tilde{\rho}}$ and $V_{\rho}$ match exactly when $\tilde{\rho}$ and $\rho$ match.

Definition 4.17 (matching representations for the same coefficient field). Suppose $\tilde{\rho}: R_{E / F} \boldsymbol{H} \rtimes \Gamma_{E / F} \rightarrow R_{E / F} \mathrm{GL}\left(V_{\tilde{\rho}}\right)$ and $\rho: \boldsymbol{H} \rightarrow \mathrm{GL}\left(V_{\rho}\right)$ are representations of algebraic groups over $F$. Let $\iota: E \hookrightarrow \mathbb{C}$ be a complex embedding. We say that $\tilde{\rho} \iota$-matches $\rho$ if

$$
\tilde{\rho}_{\iota}=\otimes_{\iota^{\prime} \mid \iota} \rho_{\iota},
$$

with $\Gamma_{E / F}$ acting by permutations on the set $\left\{\iota^{\prime} \mid \iota \circ i_{E}\right\}$. When no confusion will result, we abbreviate "l-match" to "match". For local systems $V_{\tilde{\rho}}$ of $E$-vector spaces 
and $V_{\rho}$ of $F$-vector spaces arising in the manner of Section 2B, we say that $V_{\tilde{\rho}}$ and $V_{\rho}$ match exactly when $\tilde{\rho}$ and $\rho$ match.

Main examples of matching representations. (a) Let $\rho: \boldsymbol{H} \rightarrow R_{N / F} \mathrm{GL}(V)$ be any representation over $F$. Suppose that $N$ is a Galois closure of $F$ containing $E$. For any $F$-algebra $A$, the representation $\rho$ induces a homomorphism

$$
\begin{aligned}
& \boldsymbol{H}\left(A \otimes_{F} E\right) \rightarrow \mathrm{GL}\left(\left(V \otimes_{F} A\right) \otimes_{F} E \otimes_{F} N\right) \\
& \rightarrow \prod_{i} \mathrm{GL}\left(\left(V \otimes_{F} A\right) \otimes_{F} N\right) \rightarrow \mathrm{GL}\left(\bigotimes_{i}\left(V \otimes_{F} A\right) \otimes_{F} N\right)
\end{aligned}
$$

where the product runs over all $i$ for which $F \stackrel{i_{E}}{\longrightarrow} E \stackrel{i}{\longrightarrow} N$ and $i \circ i_{E}=i_{N}$ where $i_{E}$ and $i_{N}$ are the embeddings defining $E / F$ and $N / F$. The second map above is induced by the ring map

$$
A \otimes_{F} E \otimes_{F} N \rightarrow A \otimes_{F} N, \quad a \otimes_{F} e \otimes_{F} n \mapsto\left(a \otimes_{F} i(e) \cdot n\right)_{i} .
$$

The above homomorphisms are functorial in $A$ and so define a map

$$
R_{E / F} \boldsymbol{H} \rightarrow R_{F / N} \mathrm{GL}\left(\bigotimes_{i} V\right)
$$

This map extends naturally to

$$
\tilde{\rho}: R_{E / F} \boldsymbol{H} \rtimes \Gamma_{E / F} \rightarrow R_{F / N} \mathrm{GL}\left(\bigotimes_{i} V\right),
$$

where $\Gamma_{E / F}$ acts by permuting the embeddings $i$. For every complex embedding $\iota: N \hookrightarrow \mathbb{C}$, the representations $\rho$ and $\tilde{\rho} \iota$-match.

(b) Let $\rho: \boldsymbol{H} \rightarrow \mathrm{GL}(V)$ be any representation over $F$. There is a natural map

$$
\boldsymbol{H}\left(A \otimes_{F} E\right) \rtimes \Gamma_{E / F} \rightarrow \mathrm{GL}\left(V \otimes_{F} A \otimes_{F} E\right)
$$

given by composing $\rho$ on $\left(A \otimes_{F} E\right)$-valued points with the $F$-algebra homomorphism

$$
A \otimes_{F} E \rightarrow A \otimes_{F} E, \quad a \otimes_{F} e \mapsto a \otimes_{F} \sigma(e)
$$

for each $\sigma \in \Gamma_{E / F}$. This collection of maps is functorial in $F$-algebras $A$ and so defines an algebraic group homomorphism over $F$,

$$
\tilde{\rho}: R_{E / F} \boldsymbol{H} \rtimes \Gamma_{E / F} \rightarrow R_{E / F} \mathrm{GL}(V) .
$$

For every complex embedding $\iota: E \hookrightarrow \mathbb{C}$, the representations $\rho$ and $\tilde{\rho} \iota$-match. 


\section{D. An abstract matching theorem.}

4D1. Notational setup for the matching theorem. Let $\mathcal{M}_{\mathcal{U}}=\boldsymbol{G}(E) \backslash \boldsymbol{G}\left(\mathbb{A}_{E}\right) / \mathcal{K U}$, where $\mathcal{K}, \mathcal{U}$ are chosen to be $\Gamma_{E / F}$-stable. This locally symmetric space is acted on by $\Gamma_{E / F}=\langle\sigma\rangle$, which is cyclic of prime degree $p$, by isometries. Similarly, for compact open $U \subset \boldsymbol{G}\left(\mathbb{A}_{F}^{\text {fin }}\right)$ and maximal compact $K$, we let $M_{U}=\boldsymbol{G}(E) \backslash \boldsymbol{G}\left(\mathbb{A}_{F}\right) / K U$. Let $\rho: \boldsymbol{G} \rightarrow R_{N / F} \mathrm{GL}\left(V_{\rho}\right)$ and $\tilde{\rho}: R_{E / F} \boldsymbol{G} \rightarrow R_{N / F} \mathrm{GL}\left(V_{\tilde{\rho}}\right)$ be matching representations and let $\iota: N \hookrightarrow \mathbb{C}$ be a complex embedding.

For any compact group $J$, we let $d j$ denote its volume-1 Haar measure.

4D2. Statement and proof of the matching theorem.

Theorem 4.18. Assume that

$$
\prod \mathbf{1}_{\mathcal{U}_{v}} d u_{v}=\mathbf{1}_{\mathcal{U}} d \tilde{u} \quad \text { and } \prod m_{v}=\sum_{\Pi(\mathcal{U})} c_{U} \mathbf{1}_{U} d u
$$

are trace-matching test functions (see Definition 4.13), where $\Pi(\mathcal{U})$ is a finite set of compact open subgroups of $\boldsymbol{G}\left(\mathbb{A}_{F}^{\text {fin }}\right)$. Let $\tilde{\rho}$ and $\rho$ be matching representations of $R_{E / F} \boldsymbol{G}_{E} \rtimes \Gamma_{E / F}$ and $\boldsymbol{G}$ (defined in Section $4 C$ ). Then

$\zeta_{j, \mathcal{M}_{\mathcal{U}}, \mathcal{L}_{\tilde{\rho}_{l}}, \sigma}(s)= \begin{cases}p(-1)^{a^{2}(p-1)} \sum c_{U} \zeta_{a, M_{U}, L_{\rho_{l}}}(s) \cdot p^{-s} & \text { if } j=p a, \\ 0 & \text { if } j \neq 0(\bmod p) .\end{cases}$

Proof. Because the groups $\boldsymbol{G}$ and $R_{E / F} \boldsymbol{G}$ satisfy multiplicity one, we can rewrite the above zeta functions from equations (6) and (7) as

$$
\begin{gathered}
\zeta_{j, \mathcal{M}_{\mathcal{U}}, \mathcal{L}_{\tilde{\rho}_{l}}, \sigma}(s)=\sum_{\lambda} \lambda^{-s} \sum_{\substack{\tilde{\pi} \text { essential } \\
\lambda_{\tilde{\rho}_{l}}-\lambda_{\tilde{\pi}_{\infty}}=\lambda}}\left\langle\tilde{\pi}_{\text {fin }}^{\mathcal{U}}\right\rangle \cdot\left\langle\operatorname{Hom}_{\mathcal{K}}\left(\wedge^{j} \tilde{\mathfrak{p}}, \tilde{\pi}_{\infty} \otimes \tilde{\rho}_{l}\right)\right\rangle, \\
\zeta_{j, M_{U}, L_{\rho_{l}}}(s)=\sum_{\lambda} \lambda^{-s} \sum_{\substack{\pi \\
\lambda_{\rho_{l}}-\lambda_{\pi_{\infty}}=\lambda}} \operatorname{dim} \pi_{\text {fin }}^{U} \cdot \operatorname{dim}\left(\operatorname{Hom}_{K}\left(\wedge^{j} \mathfrak{p}, \pi_{\infty} \otimes \rho_{l}\right)\right) .
\end{gathered}
$$

We now discuss matching for the multiplicities, the Casimir eigenvalues, and the $(\mathfrak{g}, K)$-cochain groups in turn.

By Lemma 4.15, for any matching pair of an essential representation $\tilde{\pi}$ of $\boldsymbol{G}\left(\mathbb{A}_{E}\right) \rtimes \Gamma_{E / F}$ and an irreducible representation $\pi$ of $\boldsymbol{G}\left(\mathbb{A}_{F}\right)$, there is an equality

$$
\left\langle\tilde{\pi}_{\text {fin }}^{\mathcal{U}}\right\rangle=\sum_{U} c_{U} \operatorname{dim} \pi_{\text {fin }}^{U} .
$$

We can relate the Casimir eigenvalues of $\tilde{\pi}_{\infty}$ and $\pi_{\infty}$ by being more explicit about the relationship between $\tilde{\pi}_{\infty}$ and $\pi_{\infty}$. Because $[E: F]$ is odd, we have $E_{\mathbb{R}} \cong\left(F_{\mathbb{R}}\right)^{p}$. A choice of isomorphism determines an isomorphism $\boldsymbol{G}\left(E_{\mathbb{R}}\right)=\boldsymbol{G}\left(F_{\mathbb{R}}\right)^{p}$. Langlands $[1980, \S 8]$ calculates that the representation $\pi_{\infty}$ corresponds to the representation $\tilde{\pi}_{\infty}=\pi_{\infty}^{\otimes p}$ of $\boldsymbol{G}\left(E_{\mathbb{R}}\right) \rtimes \Gamma_{E / F}$ equipped with intertwining isomorphism given by 
a cyclic shift. Similarly, $\tilde{\rho}_{l}$ is isomorphic to $\rho_{l}^{\bigotimes p}$ with intertwining isomorphism given by the same cyclic shift. Therefore,

$$
\lambda_{\tilde{\rho}_{l}}-\lambda_{\tilde{\pi}_{\infty}}=p\left(\lambda_{\rho_{l}}-\lambda_{\pi_{\infty}}\right) .
$$

Finally, note that because $\tilde{\pi}_{\infty} \otimes \tilde{\rho}_{\iota} \cong \pi_{\infty}^{\otimes p} \otimes \rho_{l}^{\otimes p}$ the $(\mathfrak{g}, K)$-chain complexes obey a "Künneth relationship",

$$
\operatorname{Hom}_{\mathcal{K}}\left(\wedge^{\bullet} \tilde{\mathfrak{p}}, \tilde{\pi}_{\infty} \otimes \tilde{\rho}_{l}\right)=\operatorname{Hom}_{K}\left(\wedge^{\bullet} \mathfrak{p}, \pi_{\infty} \otimes \rho_{l}\right)^{\otimes p} \quad(\text { graded tensor product })
$$

(see [Borel and Wallach 2000, Chapter I, §1.3]) which is equivariant for the cyclic shift on the right and the $\sigma$ action on the left. Exactly as in our calculations from [Lipnowski 2014, Proposition 5.1], it follows that

$$
\begin{aligned}
& \left\langle\operatorname{Hom}_{\mathcal{K}}\left(\wedge^{j} \tilde{\mathfrak{p}}, \tilde{\pi}_{\infty} \otimes \tilde{\rho}_{l}\right)\right\rangle \\
& = \begin{cases}(-1)^{a^{2}(p-1)} \cdot \operatorname{dim}\left(\operatorname{Hom}_{K}\left(\wedge^{a} \mathfrak{p}, \pi_{\infty} \otimes \rho_{l}\right)\right) & \text { if } j=p a, \\
0 & \text { if } j \text { is not a multiple of } p .\end{cases}
\end{aligned}
$$

By Langlands' formulation of base change (see Corollary 4.10), there is a bijection between irreducible subrepresentations $\pi \subset W$ of $\boldsymbol{G}\left(\mathbb{A}_{F}\right)$ and essential representations $\tilde{\pi} \subset \mathcal{W}$ of $\boldsymbol{G}\left(\mathbb{A}_{E}\right) \rtimes \Gamma_{E / F}$. Multiplying (16), (17) $)^{-s}$ and (18) together and summing over all subrepresentations $\pi$ of $W$ therefore gives the result.

An immediate corollary concerning analytic torsion is as follows:

\section{Corollary 4.19.}

$\log \tau_{\sigma}\left(\mathcal{M}_{\mathcal{U}}, \mathcal{L}_{\tilde{\rho}_{l}}\right)=p\left(\sum c_{U} \log \tau\left(M_{U}, V_{\rho_{l}}\right)\right)-p \log (p)\left(\sum_{j}(-1)^{j} j \cdot \zeta_{j, M_{U}, L_{\rho_{l}}}(0)\right)$.

Remark 4.20. The second summand on the right-hand side is a red herring. It would disappear by scaling the metric on $M_{U}$ by $p$. In particular, if $L_{\rho, \mathbb{Q}}$ is acyclic, the analytic torsion is metric independent and so the second summand automatically vanishes.

\section{Matching at places where $E / F$ is unramified}

We recall our notational setup: let $\boldsymbol{G}$ be the adjoint group of the group of units of a quaternion algebra $D$ over a number field $F$. Say $E / F$ is a cyclic Galois extension of prime degree $p$. The Galois group $\Gamma_{E / F}=\langle\sigma\rangle$ acts on $\boldsymbol{G}\left(\mathbb{A}_{E}\right)$.

The goal of this section is to describe instances of local trace-matching (see Section 4B1) at places $v$ for which $E_{v} / F_{v}$ is unramified (see, in particular, Theorem 5.4); this will enable us to prove relationships between twisted analytic torsion on locally symmetric spaces related by base change — see Theorem 4.18. 
5A. Parahoric level structure. Throughout this section, assume that $E / F$ is everywhere unramified.

The argument for proving Theorem 4.18 and Corollary 4.19 which relates $\tau_{\sigma}\left(\mathcal{L}_{\tilde{\rho}_{l}}\right)$ to $\tau\left(L_{\rho_{l}}\right)$ hinges on the fact that $\mathbf{1}_{\mathcal{U}} d \tilde{u}$ and $m=\mathbf{1}_{U^{\Sigma}} d u^{\Sigma} \times \prod_{v \in \Sigma} m_{v}$ are matching test functions, for an appropriate finite set of nonarchimedean places $\Sigma$ of $F$ and corresponding measures $m_{v}$ on $\boldsymbol{G}\left(F_{v}\right)$. We introduce a type of level structure for which we will be able to prove such a matching theorem.

Definition 5.1 (parahoric level structure). A compact open subgroup $\mathcal{U}_{v} \subset \boldsymbol{G}\left(E_{v}\right)$ is called locally parahoric if it satisfies the following conditions:

- If $D$ is ramified at $v$, then $\mathcal{U}_{v}$ should equal the image of the units of the maximal order of $D_{v}$ in $\boldsymbol{G}\left(E_{v}\right)$.

- Suppose $E_{v}$ is split and $D$ is unramified at $v$. Identify $\boldsymbol{G}\left(E_{v}\right)=\boldsymbol{G}\left(F_{v}\right)^{p}$. Then $\mathcal{U}_{v}=U_{v}^{p}$ for an arbitrary compact open $U_{v} \subset \boldsymbol{G}\left(F_{v}\right)$.

- Say $E_{v} / F_{v}$ is unramified and cyclic of degree $p$, and say $D$ is unramified at $v$. The tree of $\boldsymbol{G} / F_{v}$ injects into the tree of $\boldsymbol{G} / E_{v}$ with image identified as the Galois-invariants. We insist that $\mathcal{U}_{v}=J$, where $J$ is the pointwise stabilizer in $\boldsymbol{G}\left(E_{v}\right)$ of either a vertex or an edge of the tree of $\boldsymbol{G} / F_{v}$.

If $\mathcal{U}=\prod \mathcal{U}_{v}$ is locally parahoric at all places $v$ of $F$, then we call $\mathcal{U}$ (globally) parahoric. If $\mathcal{U}$ is locally parahoric for all $v \notin \Sigma$, then we call $\mathcal{U}$ parahoric outside $\Sigma$.

Remark 5.2. The above definition does not apply at places $v$ where $E_{v} / F_{v}$ is ramified. We extend the definition of parahoric level structure, for places $v$ where $E_{v} / F_{v}$ is tamely ramified, in Definition 6.16.

To every parahoric level structure $\mathcal{U} \subset \boldsymbol{G}\left(\mathbb{A}_{E}^{\text {fin }}\right)$, we associate a matching level structure $U \subset \boldsymbol{G}\left(\mathbb{A}_{F}^{\mathrm{fin}}\right)$.

Definition 5.3. Let $\mathcal{U}_{v} \subset \boldsymbol{G}\left(E_{v}\right)$ be parahoric at $v$. We say that $U_{v}$ is associated to $\mathcal{U}_{v}$ if $U_{v}=\mathcal{U}_{v} \cap \boldsymbol{G}\left(F_{v}\right)$. Specifically:

- Suppose $D_{v}$ is ramified. We require that $U_{v}$ equals the image of the units of the maximal order of $D_{v}$ in $\boldsymbol{G}\left(F_{v}\right)$.

- Suppose that $E_{v}$ is split. Then $\mathcal{U}_{v}=\left(U_{v}^{\prime}\right)^{p}$ for some $U_{v}^{\prime} \subset \boldsymbol{G}\left(F_{v}\right)$, and we require that $U_{v}=U_{v}^{\prime}$.

- Suppose that $E_{v} / F_{v}$ is an unramified field extension and that $D_{v}$ is unramified. Then $\mathcal{U}_{v}$ equals the pointwise stabilizer in $\boldsymbol{G}\left(E_{v}\right)$ of some simplex of the tree of $\boldsymbol{G} / F_{v}$, viewed as a subset of the tree of $\boldsymbol{G} / E_{v}$. We require that $U_{v}$ be the stabilizer of that same simplex in $\boldsymbol{G}\left(F_{v}\right)$.

We say that $U=\prod U_{v}$ is associated to $\mathcal{U}=\prod U_{v}$ if $U_{v}$ is associated to $\mathcal{U}_{v}$ for all places $v$. 
With these notions in hand, we now throw the kitchen sink at the issue of matching $\mathbf{1}_{\mathcal{U}} d \tilde{u} \leftrightarrow \mathbf{1}_{U} d u$.

Theorem 5.4 (Kottwitz). Let $U_{v}$ be associated to a parahoric level structure $\mathcal{U}_{v}$ at $v$. Then the test functions $\mathbf{1}_{\mathcal{U}_{v}}$ d $\tilde{u}_{v}$ and $\mathbf{1}_{U_{v}} d u_{v}$ geometrically match, where d $\tilde{u}_{v}$ and $d u_{v}$ are volume-1 Haar measures. In particular, $\mathbf{1}_{\mathcal{U}_{v}} d \tilde{u}_{v}$ and $\mathbf{1}_{U_{v}} d u_{v}$ are trace-matching (see Definition 4.13).

Proof. Kottwitz [1986a] proves that

$$
\mathrm{SO}_{\delta, \sigma}\left(\mathbf{1}_{\mathcal{U}_{v}} d \tilde{u}_{v}\right)=\operatorname{SO}_{N \delta}\left(\mathbf{1}_{U_{v}} d u_{v}\right)
$$

for any regular semisimple $\delta \in \boldsymbol{G}\left(E_{v}\right)$. Here SO denotes the stable orbital integral, whose definition is given in [Kottwitz 1986a]. Let $c$ be a $\boldsymbol{G}\left(F_{v}\right)$-conjugacy class in $\boldsymbol{G}\left(F_{v}\right)$. Suffice it to say that if

$$
\begin{array}{r}
\left\{\boldsymbol{G}\left(\bar{F}_{v}\right) \text { conjugacy class of } c\right\} \cap \boldsymbol{G}\left(F_{v}\right)=c, \\
\left\{\boldsymbol{G}\left(\bar{E}_{v}\right) \sigma \text {-twisted conjugacy class of } c^{\prime}\right\} \cap \boldsymbol{G}\left(E_{v}\right)=c^{\prime}
\end{array}
$$

for every regular semisimple conjugacy class $c$ and regular semisimple $\sigma$-twisted conjugacy class $c^{\prime}$, then $\mathrm{SO}_{\delta, \sigma}=O_{\delta, \sigma}$ and $\mathrm{SO}_{\gamma}=O_{\gamma}$.

Corollary 4.5 shows that, if $\boldsymbol{G}$ is the adjoint group of the units of a quaternion algebra over $F_{v}$, then $\boldsymbol{G}\left(\bar{F}_{v}\right)$-conjugacy collapses to $\boldsymbol{G}\left(F_{v}\right)$-conjugacy and $\boldsymbol{G}\left(\bar{E}_{v}\right) \sigma$-conjugacy collapses to $\boldsymbol{G}\left(E_{v}\right) \sigma$-conjugacy. Thus, $\mathbf{1}_{\mathcal{U}_{v}} d \tilde{u}_{v}$ and $\mathbf{1}_{U_{v}} d u_{v}$ match, as claimed.

\section{Matching at places where $E / F$ is tamely ramified}

To broaden the applicability of Section $4 \mathrm{D}$, we need to prove matching theorems at places $v$ where $E_{v} / F_{v}$ is ramified. We assume throughout this chapter that $E_{v} / F_{v}$ is tamely ramified. Our immediate goals:

(1) Characterize all (possibly ramified) representations $\pi$ of $\operatorname{PGL}_{2}\left(F_{v}\right)$ for which $\mathrm{BC}(\pi)$ is unramified.

(2) Find a test function on $f d g$ on $\mathrm{PGL}_{2}\left(F_{v}\right)$ which trace-matches $\mathbf{1}_{\mathrm{PGL}_{2}\left(E_{v}\right)} d \tilde{g}$.

Characterizing those representations as in (1) will be relatively straightforward; this is done in Section 6A. We will say that representations as in (1) are $E_{v}$-potentially unramified. For (2), the main content is an analysis of $J$-fixed vectors in principal series representations for various compact open subgroups $J \subset \mathrm{PGL}_{2}\left(O_{F}\right)$; this is accomplished in Section 6C3. A test function which serves as an indicator function for $E_{v}$-potentially unramified representations is constructed in Theorem 6.15. 
Notation for local fields. To ease notation in what follows, we will denote $E_{v} / F_{v}$ by $E / F$. So for the remainder of this chapter, $E / F$ will denote a tamely ramified, cyclic Galois extension of local fields, not an extension of global fields.

6A. Tamely ramified and E-potentially unramified Langlands parameters. Fix a rational prime $\ell$ not equal to the residue characteristic of $F$. We want to characterize continuous representations

$$
\sigma: W_{F} \rightarrow \mathrm{SL}_{2}\left(\overline{\mathbb{Q}_{\ell}}\right)
$$

such that $\left.\sigma\right|_{W_{E}}$ is unramified. Since $E / F$ is tamely ramified, such representations $\sigma$ are tamely ramified. But the tame Weil group has the very simple description

$$
W_{F}^{\text {tame }}=I_{F}^{\text {tame }} \rtimes\langle f\rangle,
$$

where $I_{F}^{\text {tame }}$ is procyclic and generated by a single element $u$, and $f$ is any lift of the arithmetic Frobenius. The generators $f$ and $u$ satisfy the single relation

$$
f u f^{-1}=u^{q}, \quad \text { where } q=\# k_{F} .
$$

Remark 6.1. Because $\Gamma_{E / F}$ is an abelian quotient of $W_{F}^{\text {tame }}$ of prime order $p$, it follows that $p \mid(q-1)$.

Lemma 6.2. All E-potentially unramified Langlands parameters are either unramified or conjugate to

$$
\sigma=\sigma_{a, \zeta}:=f \mapsto\left(\begin{array}{cc}
a & 0 \\
0 & a^{-1}
\end{array}\right), \quad u \mapsto\left(\begin{array}{cc}
\zeta & 0 \\
0 & \zeta^{-1}
\end{array}\right)
$$

for $a, \zeta \in \overline{\mathbb{Q}}_{\ell} \times$ and $\zeta^{p}=1$.

Proof. Note that $I_{F}^{\text {tame }} / I_{E}^{\text {tame }}$ equals $\Gamma_{E / F}$, which is a cyclic group of order $p$. Thus, $I_{E}^{\text {tame }}$ must be the unique closed subgroup of index $p$ in $I_{F}^{\text {tame }}$. In particular, $u^{p} \in I_{E}^{\text {tame }}$. It follows that if $\sigma(f)=A, \sigma(u)=B$, then

$$
A B A^{-1}=B^{q} \text { and } \quad B^{p}=1 .
$$

After conjugation if necessary, we may suppose that $B=\left(\begin{array}{cc}\zeta & 0 \\ 0 & \zeta^{-1}\end{array}\right)$, where $\zeta^{p}=1$.

Assume that $\zeta \neq 1$, and let $A=\left(\begin{array}{ll}a & b \\ c & d\end{array}\right)$. Using the fact that $A B A^{-1}=B^{q}$ and that $\zeta^{q-1}=1$, we see that $b=c=0$. These give rise to the Langlands parameters

$$
\sigma=\sigma_{a, \zeta}:=f \mapsto\left(\begin{array}{cc}
a & 0 \\
0 & a^{-1}
\end{array}\right), \quad u \mapsto\left(\begin{array}{cc}
\zeta & 0 \\
0 & \zeta^{-1}
\end{array}\right) .
$$

These parameters correspond, by the local Langlands correspondence, to the representations $I_{s, \chi}:=\operatorname{Ind}_{B}^{G}\left(|\cdot|^{s} \cdot \chi\right)$ where $\chi$ is a nontrivial character of $F^{\times} / N\left(E^{\times}\right)$.

If $\zeta=1$, the resulting parameter corresponds to an unramified representation. 
6B. A closer look at local base change. Let $E / F$ be a tamely ramified extension of local fields. The goal of this section is to construct a smooth, compactly supported test measure $m_{E \text {,ur }}$ on $\mathrm{PGL}_{2}(F)$ such that $\mathbf{1}_{\mathrm{PGL}_{2}\left(O_{E}\right)} d \tilde{k}$ trace-matches $m_{E \text {,ur }}$ (see Definition 4.13).

In Section 6B1, we explain how to recover the matching of orbital integrals in a more representation theoretic manner. In Section 6B2, we discuss the action of $\Gamma_{E / F}$ on the $\mathrm{PGL}_{2}\left(O_{E}\right)$-fixed vectors of a representation of $\mathrm{PGL}_{2}(E)$ of the form $\mathrm{BC}(\pi)$.

6B1. Strategy for proving the local base change trace identity. Recall the following definition from Section 4 on base change:

Definition 6.3. A representation $\tilde{\pi}$ of $\mathrm{PGL}_{2}(E) \rtimes\langle\sigma\rangle$ matches the representation $\pi$ of $\mathrm{PGL}_{2}(F)$ if the following equality of traces holds:

$$
\operatorname{tr}\{\tilde{\pi}(\sigma) \tilde{\pi}(\tilde{f} d \tilde{g})\}=\operatorname{tr}\{\pi(f d g)\}
$$

for every pair of matching test functions $\tilde{f} d \tilde{g} \in \mathrm{SM}_{c}(\boldsymbol{G}(E)), f d g \in \mathrm{SM}_{c}(\boldsymbol{G}(F))$. We write $\tilde{\pi}=\mathrm{BC}(\pi)$.

Remark 6.4. The intertwining isomorphism $\tilde{\pi}(\sigma): \pi \rightarrow \pi^{\sigma}$ is a priori only welldefined up to a $p$-th root of unity. However, the equality of traces in the above definition uniquely determines $\tilde{\pi}(\sigma)$.

For the particular choices of test functions $\tilde{f}_{0} d \tilde{g}_{0}$ and $f_{0} d g_{0}$ that we will make in the sequel, rather than proving that (19) holds by demonstrating an equality of orbital integrals, we prove it directly by using explicitly understood features of local base change for $\mathrm{PGL}_{2}(F)$.

6B2. Action of $\sigma$ on $\mathrm{PGL}_{2}\left(O_{E}\right)$-fixed vectors. Let $\mu$ be the inflation to the upper triangular Borel subgroup $B \subset \mathrm{PGL}_{2}(F)$ of a character $T \rightarrow \mathbb{C}^{\times}$, where $T$ is the diagonal torus of $\mathrm{PGL}_{2}(F)$, and let $\delta$ denote the modulus character of $B$. We let $\left(\rho_{\mu}, I_{\mu}\right)$ denote the normalized principal series representation, i.e., the space of locally constant functions $f: \mathrm{PGL}_{2}(F) \rightarrow \mathbb{C}$ which transform by the rule $f(b g)=\mu(b) \delta(b)^{1 / 2} f(g)$ where $\rho_{\mu}$ acts by right translation.

We define $I_{\tilde{\mu}}$ similarly for characters of the upper triangular Borel subgroup $\widetilde{B} \subset \operatorname{PGL}_{2}(E)$. If $\tilde{\mu}^{\sigma}=\tilde{\mu}$, then we extend $I_{\tilde{\mu}}$ to a representation of $\operatorname{PGL}_{2}(E) \rtimes \Gamma_{E / F}$ by the formula

$$
\rho_{\tilde{\mu}}(\sigma) f(g)=f\left(g^{\sigma^{-1}}\right) .
$$

Proposition 6.5 [Langlands 1980, Corollary 7.3]. Let $N: E^{\times} \rightarrow F^{\times}$denote the norm map. The representations $I_{\mu \circ N}$ and $I_{\mu}$ match. That is, for any pair $\tilde{f} d \tilde{g}$ and $f d g$ of matching test functions on $\mathrm{PGL}_{2}(E)$ and $\mathrm{PGL}_{2}(F)$ respectively, there is an equality

$$
\operatorname{tr}\left\{\rho_{\mu \circ N}(\sigma) \rho(\tilde{f} d \tilde{g})\right\}=\operatorname{tr}\left\{\rho_{\mu}(f d g)\right\}
$$


Corollary 6.6. For every irreducible admissible representation $\pi$ of $\mathrm{PGL}_{2}(F)$, there is an equality

$$
\begin{aligned}
\operatorname{tr}\{\mathrm{BC}(\pi)(\sigma) \mathrm{BC}(\pi) & \left.\left(\mathbf{1}_{\mathrm{PGL}_{2}\left(O_{E}\right)} d \tilde{k}\right)\right\} \\
& =\operatorname{tr}\left\{\mathrm{BC}(\pi)\left(\mathbf{1}_{\mathrm{PGL}_{2}\left(O_{E}\right)} d \tilde{k}\right)\right\}= \begin{cases}1 & \text { if } \mathrm{BC}(\pi) \text { is unramified } \\
0 & \text { otherwise. }\end{cases}
\end{aligned}
$$

Proof. The claim is vacuously true if $\mathrm{BC}(\pi)$ is ramified. If it is unramified, then it can be embedded in an unramified principal series representation $V$; indeed, any representation with an Iwahori fixed vector can be embedded into an unramified principal series representation.

If $V$ is irreducible, then $\mathrm{BC}(\pi)=V$. But Proposition 6.5 shows that for such principal series representations, in the image of base change, the action of $\Gamma_{E / F}$ on the $\mathrm{PGL}_{2}\left(O_{E}\right)$-fixed vector is trivial.

If $V$ is reducible, then $\mathrm{BC}(\pi)$ is either Steinberg, which is ramified, or the trivial representation. One readily checks that the trivial representations of $\operatorname{PGL}_{2}(F)$ and $\mathrm{PGL}_{2}(E) \rtimes \Gamma_{E / F}$ match because each has character given by the constant function 1 . Therefore, $\Gamma_{E / F}$ again acts trivially on the $\mathrm{PGL}_{2}\left(O_{E}\right)$-fixed vector of $\mathrm{BC}(\pi)$.

\section{C. Constructing an indicator test function for E-potentially unramified repre-} sentations. According to Corollary 6.6, finding a function on $\mathrm{PGL}_{2}(F)$ which trace-matches the volume-1 Haar measure on $\mathrm{PGL}_{2}\left(O_{E}\right)$ is equivalent to finding a test measure $m_{E \text {,ur }}$ on $\mathrm{PGL}_{2}(F)$ satisfying

$$
\operatorname{tr} \pi\left(m_{E, \mathrm{ur}}\right)= \begin{cases}1 & \text { if } \pi \text { is } E \text {-potentially unramified, } \\ 0 & \text { otherwise. }\end{cases}
$$

The Langlands parameters for all $E$-potentially unramified representations are, in particular, tamely ramified. By the local Langlands correspondence, they therefore have Iwahori fixed vectors. Therefore, a natural place to start constructing such a test measure $m_{E \text {, ur }}$ is to modify the measure $\mathbf{1}_{J} d j$ for congruence subgroups of Iwahori subgroups.

6C1. Fixed vectors for congruence subgroups of Iwahori subgroups of $\mathrm{PGL}_{2}(F)$. Let $\boldsymbol{B} \subset \mathrm{PGL}_{2} / O_{F}$ be a Borel subgroup and $N$ its unipotent radical, and let $\boldsymbol{T}=\boldsymbol{B} / \boldsymbol{N}$. Let $k_{F}$ denote the residue field of $O_{F}$, and let $C$ be any subgroup of $\boldsymbol{T}\left(k_{F}\right)$. Let $I_{C} \subset \mathrm{PGL}_{2}\left(O_{F}\right)$ denote the preimage of $C$ under the reduction map. The subgroup $I_{1}$ is one such example.

6C2. Supercuspidal representations have no $I_{C}$-fixed vectors. We quote the following theorem:

Proposition 6.7 [Bushnell and Henniart 2006, §14.3]. If $V$ is a supercuspidal representation of $\mathrm{PGL}_{2}(F)$, then $V^{I_{1}}=0$. In particular, $V^{I_{C}}=0$ for any $C$ as above. 
6C3. $I_{C}$-fixed vectors for principal series representations of $\mathrm{PGL}_{2}(F)$. Suppose $B=\boldsymbol{B}(F), N=N(F), T=\boldsymbol{T}(F), G=\mathrm{PGL}_{2}(F)$. We prove a simple lemma concerning the space of $J$-fixed vectors, for an arbitrary compact open $J$, in principal series representations $I_{\chi}=\operatorname{Ind}_{B}^{G} \chi$, where $\chi: B \rightarrow \mathbb{C}^{\times}$is the inflation of a character of $T$.

Lemma 6.8. The dimension of the space of J-fixed vectors $\left(I_{\chi}\right)^{J}$ equals the number of double cosets $g \in B \backslash G / J$ for which $\left.\chi\right|_{B \cap g g^{-1}}=1$.

Proof. Let $V=I_{\chi}$. If $f \in V^{J}$, then $f(b g j)=\chi(b) f(g)$ for $b \in B, j \in J$. In order for this equality to hold for all $b \in B, j \in J$, it is necessary and sufficient that either $\left.\chi\right|_{B \cap g J g^{-1}}=1$ or $f(g)=0$. Therefore, $f \in V^{J}$ is uniquely specified by its values on those double cosets $g$ for which $\chi_{B \cap g g^{-1}}=1$.

By choosing $J=I_{C}$ well, we can essentially isolate all those $E$-potentially unramified representations, i.e., those whose Langlands parameters were classified in Section 6A.

Corollary 6.9. Let $T_{C}=\left\{t \in \boldsymbol{T}\left(O_{F}\right): t(\bmod \pi) \in C \subset \boldsymbol{T}\left(k_{F}\right)\right\}$ and let $J=I_{C}$. Then

$$
\operatorname{dim}\left(I_{\chi}\right)^{J}=\left\{\begin{array}{lc}
2 & \text { if }\left.\chi\right|_{T_{C}}=1 \\
0 & \text { otherwise }
\end{array}\right.
$$

Proof. Observe that the elements

$$
1 \text { and } w=\left(\begin{array}{ll}
0 & 1 \\
1 & 0
\end{array}\right)
$$

form a full set of coset representatives for $B \backslash G / J$. But we readily compute that the image of both $B \cap w J w^{-1}$ and $B \cap J$ in $T$ is $T_{C}$. The result then follows immediately by Lemma 6.8 .

Corollary 6.10. Let

$$
C_{0}=\left\{\left(\begin{array}{rr}
a^{p} & 0 \\
0 & 1
\end{array}\right): a \in k_{F}^{\times}\right\}
$$

Then

$$
\operatorname{dim}\left(I_{\chi}\right)^{I_{C_{0}}}= \begin{cases}2 & \text { if }\left.\chi\right|_{N\left(O_{E}^{\times}\right)}=1 \\ 0 & \text { otherwise }\end{cases}
$$

Remark 6.11. As pointed out in Section 6A, the $E$-potentially unramified principal series representations are exactly those with Langlands parameters corresponding to $\chi$ for which $\left.\chi\right|_{N\left(O_{E}^{\times}\right)}=1$, as in the first case of the above corollary. 
6C4. $I_{C_{0}}$-fixed vectors in arbitrary representations.

Proposition 6.12. Let $\pi$ be an irreducible admissible representation of $\operatorname{PGL}_{2}(F)$. Then

$$
\operatorname{tr} \pi\left(1_{C_{0}} d j\right)= \begin{cases}0 & \text { if } \pi \text { is supercuspidal, } \\ 0 & \text { if } \pi=I_{\chi},\left.\chi\right|_{N\left(O_{E}^{\times}\right)} \neq 1, \\ 2 & \text { if } \pi=I_{\chi},\left.\chi\right|_{N\left(O_{E}^{\times}\right)}=1, \\ 1 & \text { if } \pi=\mathbf{1}, \\ 1 & \text { if } \pi=\mathrm{St},\end{cases}
$$

where $d j$ denotes the volume-1 Haar measure on $I_{C_{0}}$.

Proof. Irreducible admissible representations $\pi$ of $\operatorname{PGL}_{2}(F)$ are of three possible types:

- $\pi$ is supercuspidal. As discussed in Section 6C2, no supercuspidal representation has an $I_{C_{0}}$-fixed vector.

- By definition, if $\pi$ is not supercuspidal then it is a subquotient of a principal series representation. All of the principal series representations $I_{\chi}$, where $\chi \neq|\cdot|$ and 1, are irreducible. As explained in Section 6C3, such representations have a 2-dimensional space of $I_{C_{0}}$-fixed vectors if $\left.\chi\right|_{N\left(O_{E}^{\times}\right)}=1$ and a 0-dimensional space of $I_{C_{0}}$-fixed vectors otherwise.

- The representations $I_{|\cdot|}$ and $I_{1}$ are reducible. In both cases, there is one trivial subquotient and another Steinberg subquotient. Both subquotients, the trivial representation and the Steinberg, have a 1-dimensional space of $I_{C_{0}}$-invariants.

The result is simply the aggregate of all of these possibilities.

6C5. Isolating 1 and St using the Euler-Poincaré test function. In his work on Tamagawa numbers, Kottwitz made use of a test function which almost isolates the Steinberg representation.

Let $C$ be a closed chamber of the building of $H=\boldsymbol{H}(F)$ for a semisimple $\boldsymbol{H}$ over $F$. For a compact open subgroup $J \subset H$, let $d g_{J}$ denote the Haar measure of $H$ assigning $J$ measure 1. Let

$$
\mathrm{EP}:=\sum_{d=0}^{\operatorname{rank} \boldsymbol{H}} \sum_{\begin{array}{c}
\text { stabilizers of } \operatorname{dim} d \\
\text { facets of } C
\end{array}}(-1)^{d} \mathbf{1}_{J} d g_{J} .
$$

Theorem 6.13 [Kottwitz 1986b]. For an irreducible unitary representation $\pi$ of $H$,

$$
\operatorname{tr} \pi(\mathrm{EP})=\chi\left(H^{\bullet}(\pi)\right)= \begin{cases}1 & \text { if } \pi=\mathbf{1}, \\ (-1)^{\operatorname{rank} \boldsymbol{H}} & \text { if } \pi=\mathrm{St}, \\ 0 & \text { otherwise. }\end{cases}
$$


Corollary 6.14. In the case of $\boldsymbol{H}=\mathrm{PGL}_{2}$, the formula from Theorem 6.13 holds for any irreducible admissible representation.

Proof. Once again, we invoke the classification of irreducible admissible representations of $\mathrm{PGL}_{2}(F)$. Any nonunitary irreducible admissible representation must be an irreducible principal series representation of the form $\pi_{s}=\left.I_{|\cdot|}\right|^{s} \chi_{0}$. But

$$
s \mapsto \operatorname{tr} \pi_{s}(\mathrm{EP})
$$

is a continuous, integer-valued function of $s$ and so must be constant and equal to $\operatorname{tr} \pi_{0}(\mathrm{EP})=0$.

We are finally able to write down an indicator test function for $E$-potentially unramified representations.

Theorem 6.15. The test function $m_{E, \text { ur }}=\frac{1}{2}\left[\mathrm{EP}+1_{C_{0}} d j\right]$ satisfies

$$
\operatorname{tr} \pi\left(m_{E, \mathrm{ur}}\right)= \begin{cases}1 & \text { if } \pi \text { is E-potentially unramified }, \\ 0 & \text { otherwise. }\end{cases}
$$

Proof. This follows immediately from Proposition 6.12 and Corollary 6.14.

6D. Definition of tame parahoric level structure and globally matching test functions. We now extend the definition of parahoric level structure to include places $v$ of $F$ for which $E_{v} / F_{v}$ is tamely ramified.

Definition 6.16. Let $E / F$ be everywhere tamely ramified. Also, assume that the set of places $v$ where $E_{v} / F_{v}$ is ramified is disjoint from the set of places where $D_{F_{v}}$ is ramified. Let $\mathcal{U}_{v} \subset \boldsymbol{G}\left(E_{v}\right)$ be a compact open subgroup. We call $\mathcal{U}_{v}$ tamely parahoric at $v$ if

- $\mathcal{U}_{v}$ is parahoric at $v$ if $E_{v} / F_{v}$ is split or unramified, and

- $\mathcal{U}_{v}=\operatorname{PGL}_{2}\left(O_{E_{v}}\right)$ if $E_{v} / F_{v}$ is tamely ramified.

We call a level structure $\mathcal{U}=\prod \mathcal{U}_{v} \subset \boldsymbol{G}\left(\mathbb{A}_{E}^{\mathrm{fin}}\right)$ (globally) tamely parahoric if $\mathcal{U}_{v}$ is tamely parahoric at $v$ for all $v$.

We now consolidate all of the preceding results of Section 6 to provide a class of examples of globally matching test functions.

Theorem 6.17. Let $E / F$ be everywhere tamely ramified, with $\Sigma=\left\{v_{1}, \ldots, v_{n}\right\}$ the places of $F$ which ramify in $E$. Let $\mathcal{U} \subset \boldsymbol{G}\left(\mathbb{A}_{E}^{\mathrm{fin}}\right)$ be a globally tamely parahoric level structure. Define $\mathcal{U}^{\Sigma}=\prod_{v \notin \Sigma} \mathcal{U}_{v}$, and let $U^{\Sigma}$ denote a matching parahoric level structure outside $\Sigma$. For $v \in \Sigma$, let

$$
m_{E_{v}, \text { ur }}=\frac{1}{2} \mathbf{1}_{K_{v}} d k_{v}+\frac{1}{2} \mathbf{1}_{K_{v}^{\prime}} d k_{v}^{\prime}-\frac{1}{2} \mathbf{1}_{I_{v}} d i_{v}+\frac{1}{2} \mathbf{1}_{I_{C_{0, v}}} d c_{0}:=\sum_{i=1}^{4} c_{v, i} d u_{v, i},
$$


where $K_{v}$ and $K_{v}^{\prime}$ are the stabilizers of two vertices in the tree for $\boldsymbol{G}\left(F_{v}\right) \cong \mathrm{PGL}_{2}\left(F_{v}\right)$; $I_{v}$ is the pointwise stabilizer of the edge between them; $I_{C_{0, v}}$ is the "preimage in $I_{v}$ of the p-th powers of the diagonal torus mod $v$ " (cf. Corollary 6.10); and dk denotes the volume-1 Haar measure for any compact group $K$. Then the test functions

$$
\mathbf{1}_{\mathcal{U}} d u \leftrightarrow \sum c_{v_{1}, i_{1}} \cdots c_{v_{n}, i_{n}} \mathbf{1}_{\prod_{j=1}^{n} U_{v_{j}, i_{j}} \times U^{\Sigma}} \prod_{j=1}^{n} d u_{v_{j}, i_{j}} \times d u^{\Sigma}
$$

trace-match (see Definition 4.13).

Proof. Theorem 6.15 shows that $m_{E_{v} \text {, ur }}$ trace-matches $\mathbf{1}_{\mathcal{U}_{v}} d \tilde{u}_{v}$ for each place $v \in \Sigma$. For $v \notin \Sigma$, let $U_{v}$ be associated to the parahoric level structure $\mathcal{U}_{v}$. Theorem 5.4 shows that $\mathbf{1}_{U_{v}} d u_{v}$ trace-matches $\mathbf{1}_{\mathcal{U}_{v}} d \tilde{u}_{v}$. Therefore,

$$
\mathbf{1}_{\mathcal{U}} d \tilde{u} \leftrightarrow \prod_{v \in \Sigma} m_{E_{v}, \mathrm{ur}} \times \mathbf{1}_{U^{\Sigma}} d u^{\Sigma}
$$

are matching test functions. The result follows after expanding the right-hand side of (20).

\section{A numerical form of base change functoriality for torsion}

In this section, we'll exhibit examples of $F$-acyclic local systems of $O_{F}$-modules on locally symmetric spaces associated to $\boldsymbol{G}$ for which the Cheeger-Müller theorems of [Lipnowski 2014, §1.7, §4] can be applied. In conjunction with the trace formula comparison proven in Section 4D, this will yield a numerical form of torsion base change functoriality.

In Section 7A, we will describe a certain class of $F$-acyclic local systems of $O_{F}$-modules over locally symmetric spaces associated to $\boldsymbol{G}$. In Section 7B, we will prove the main comparison theorem of this paper, concerning a version of "numerical functoriality".

7A. A class of local systems. We recall the construction of some local systems described in [Calegari and Venkatesh 2012, §10.1].

For any quaternion algebra $Q$ over $F$, we let $[Q]_{m}$ denote the $F$-vector space $[Q]_{m}=\operatorname{Sym}^{m} Q^{0} / \Delta \operatorname{Sym}^{m-2} Q^{0}$, where $Q^{0}$ denotes the trace zero elements of $Q$ and $\Delta$ denotes any invariant element of $\operatorname{Sym}^{2} Q^{0}$. The $F$-vector space $[Q]_{m}$ affords an $F$-linear representation of $Q^{\times} / F^{\times}$, which acts by conjugation on $Q$ and thus on the symmetric powers of $Q^{0}$.

Let $V_{a, b}=[D]_{a} \otimes[\bar{D}]_{b}$, where $\bar{D}$ denotes the quaternion algebra $D \otimes_{\sigma} F$, with $\sigma$ denoting the nontrivial automorphism of $F$. We note that $[\bar{D}]_{b}=\left[\overline{D]_{b}}\right.$.

Consider the representation $\rho_{a, b}: \boldsymbol{G} \rightarrow \mathrm{GL}\left(V_{a, b}\right)$ given by conjugation action. By the second construction outlined in Section $4 \mathrm{C}$, there is a matching representation $\tilde{\rho}_{a, b}: R_{E / F} \boldsymbol{G} \rtimes \Gamma_{E / F} \rightarrow R_{E / F} \operatorname{GL}\left(V_{a, b}\right)$. 
Suppose that $U \subset \boldsymbol{G}\left(\mathbb{A}_{F}^{\mathrm{fin}}\right)$ is compact open and $K \subset \boldsymbol{G}\left(F_{\mathbb{R}}\right)$ is a maximal compact subgroup. Suppose that $\mathcal{U} \subset \boldsymbol{G}\left(\mathbb{A}_{E}^{\text {fin }}\right)$ is compact open and $\mathcal{K} \subset \boldsymbol{G}\left(E_{\mathbb{R}}\right)$ is maximal compact, with both $\mathcal{U}$ and $\mathcal{K}$ Galois-stable. Fix an $O_{F}$-lattice $\mathcal{O} \subset \rho_{a, b}$ and an $O_{E}$-lattice $\widetilde{\mathcal{O}} \subset \tilde{\rho}_{a, b}$. Suppose that $U$ stabilizes $\mathcal{O}$ and $\mathcal{U}$ stabilizes $\widetilde{\mathcal{O}}$. By the construction of Section $2 \mathrm{~B}$, the matching representations $\rho_{a, b}$ and $\tilde{\rho}_{a, b}$ together with these data give rise to matching local systems

$$
L_{a, b} \rightarrow M_{U}=\boldsymbol{G}(F) \backslash \boldsymbol{G}\left(\mathbb{A}_{F}\right) / K U, \quad \mathcal{L}_{a, b} \rightarrow \mathcal{M}_{\mathcal{U}}=\boldsymbol{G}(E) \backslash \boldsymbol{G}\left(\mathbb{A}_{E}\right) / \mathcal{K} \mathcal{U} .
$$

For each complex embedding $\iota: E \hookrightarrow \mathbb{C}$, we let

$$
L_{a, b, l}=L_{a, b} \otimes_{\iota \circ i_{F}} \mathbb{C}, \quad \mathcal{L}_{a, b, l}=\mathcal{L}_{a, b} \otimes_{\iota} \mathbb{C},
$$

be local systems of $\mathbb{C}$-vector spaces.

7A1. Acyclicity. We recall a theorem of Borel-Wallach, specialized to our setup: Theorem 7.1 [Borel and Wallach 2000, §II, Proposition 6.12]. Let $\mathcal{L} \rightarrow \mathcal{M}_{\mathcal{U}}$ be the local system of $\mathbb{C}$-vector spaces corresponding to a complex representation $\rho: \boldsymbol{G}\left(E_{\mathbb{R}}\right)=R_{E / F} \boldsymbol{G}\left(F_{\mathbb{R}}\right) \rightarrow \operatorname{GL}(V)$. Suppose that $d \rho: \operatorname{Lie}\left(\boldsymbol{G}\left(E_{\mathbb{R}}\right)\right) \rightarrow \operatorname{End}(V)$ is not isomorphic to its twist by the Cartan involution of $\boldsymbol{G}\left(E_{\mathbb{R}}\right)$ corresponding to $\mathcal{K}$. Then $\mathcal{L} \rightarrow \mathcal{M}_{\mathcal{U}}$ is acyclic. A similar statement holds for a local system of $\mathbb{C}$-vector spaces $L \rightarrow M_{U}$ corresponding to representations of $\boldsymbol{G}\left(F_{\mathbb{R}}\right)$.

Proof. See [Bergeron and Venkatesh 2013, §4.1], wherein the stronger statement that representations $\rho$ as above give rise to "strongly acyclic families" is proven.

Corollary 7.2. The local systems $L_{a, b, l} \rightarrow M_{U}, \mathcal{L}_{a, b, l} \rightarrow \mathcal{M}_{\mathcal{U}}$ are acyclic provided that $a \neq b$.

Corollary 7.3. The $O_{E}$-module $H^{\bullet}\left(\mathcal{M}_{U}, \mathcal{L}_{a, b}\right)$ and the $O_{F}$-module $H^{\bullet}\left(M_{U}, L_{a, b}\right)$ are torsion for $a \neq b$.

7B. Numerical comparison theorem. We can finally combine our trace formula comparisons with the Cheeger-Müller theorems of [Lipnowski 2014, §1.7] to obtain a numerical comparison of Reidemeister torsion.

Theorem 7.4. Let $F$ be an imaginary quadratic field. Let $E / F$ be Galois and cyclic of odd prime degree $p$. Let $a \neq b$. Then there is an equality

$\log \mathrm{RT}_{\sigma}\left(\mathcal{M}_{\mathcal{U}}, \mathcal{L}_{a, b, l}\right)=p\left(\sum c_{v_{1}, i_{1}} \cdots c_{v_{n}, i_{n}} \log \mathrm{RT}\left(M_{\prod_{j=1}^{n} U_{v_{j}, i_{j}} \times U^{\Sigma}}, L_{a, b, l}\right)\right)$,

where the constants $c_{v, k}$ and level structures are as in Theorem 6.17.

Proof. By Theorem 6.17, the test functions

$$
\mathbf{1}_{\mathcal{U}} d u \leftrightarrow \sum c_{v_{1}, i_{1}} \cdots c_{v_{n}, i_{n}} \mathbf{1}_{\prod_{j=1}^{n} U_{v_{j}, i_{j}} \times U^{\Sigma}} \prod_{j=1}^{n} d u_{v_{j}, i_{j}} \times d u_{\Sigma}
$$


are matching. By Corollary 4.19, there is thus an equality

$$
\log \tau_{\sigma}\left(\mathcal{M}_{\mathcal{U}}, \mathcal{L}_{a, b, l}\right)=p\left(\sum c_{v_{1}, i_{1}} \cdots c_{v_{n}, i_{n}} \log \tau\left(M_{\prod_{j=1}^{n} U_{v_{j}, i_{j}} \times U^{\Sigma}}, L_{a, b, \iota}\right)\right) .
$$

By the assumption $a \neq b$, Corollary 7.2 applies and so all regulators vanish. Because $\mathcal{L}_{a, b, \iota}, L_{a, b, \iota}$ are unimodular, the twisted Bismut-Zhang theorem (see [Lipnowski 2014, Theorem 4.1]) and the untwisted Cheeger-Müller theorem of [Müller 1993] (see [Lipnowski 2014, §1.7]) apply and give

$$
\log \mathrm{RT}_{\sigma}\left(\mathcal{M}_{\mathcal{U}}, \mathcal{L}_{a, b, l}\right)=p\left(\sum c_{v_{1}, i_{1}} \cdots c_{v_{n}, i_{n}} \log \mathrm{RT}\left(M_{\prod_{j=1}^{n} U_{v_{j}, i_{j}} \times U^{\Sigma}}, L_{a, b, l}\right)\right) .
$$

The equality (21) from Theorem 7.4 is unwound into a numerical comparison between torsion cohomology groups in [Lipnowski 2014, Lemma 1.21]. A palatable corollary is as follows:

Corollary 7.5. Let $F$ be an imaginary quadratic field. Let $E / F$ be an everywhere unramified cyclic Galois extension of odd prime degree p. Let $\mathcal{U} \subset \boldsymbol{G}\left(\mathbb{A}_{E}^{\text {fin }}\right)$ be a parahoric level structure and let $U=\mathcal{U} \cap \boldsymbol{G}\left(\mathbb{A}_{F}^{\text {fin }}\right)$. Suppose $a \neq b$. Then

$$
\prod^{*}\left(\frac{\left|H^{i}\left(\mathcal{M}_{\mathcal{U}}, \mathcal{L}_{a, b}^{0}\right)^{\sigma-1}\right|}{\left|H^{i}\left(\mathcal{M}_{\mathcal{U}}, \mathcal{L}_{a, b}^{0}\right)^{P(\sigma)}\right|}\right) \sim_{p}\left(\prod^{*}\left|H^{i}\left(M_{U}, L_{a, b}^{0}\right)\right|\right)^{p},
$$

where $\sim_{p}$ denotes equality up to powers of $p$. In particular, if $\ell \neq p$ is a prime dividing $\left|H^{i}\left(M_{U}, L_{a, b}^{0}\right)\right|$ for exactly one $i$, then $\ell$ divides $\left|H^{*}\left(\mathcal{M}_{\mathcal{U}}, \mathcal{L}_{a, b}^{0}\right)\right|$.

Proof. Because $E / F$ is everywhere unramified, the right-hand side of (21) has $\mathrm{RT}\left(M_{U}, L_{a, b, \iota}\right)$ as its only summand. Summing (21) over all complex embeddings $\iota$ of $E$ and applying [Lipnowski 2014, Lemma 1.21] yields the desired result.

Remark 7.6. Empirically, the integer $\left|H^{2}\left(M_{U}, L_{a, b}^{0}\right)\right|$ tends to be supported at large sporadic primes [SSengün 2011]. On the other hand, the integers $\left|H^{i}\left(M_{U}, L_{a, b}^{0}\right)\right|$ for $i \neq 2$ provably grow at most polynomially in $\operatorname{vol}\left(M_{U}\right)$ [Bergeron and Venkatesh $2013, \S 8.6]$. Corollary 7.5 thus strongly suggests an underlying torsion base change phenomenon. See Section 1A for a more detailed discussion of torsion base change.

\section{Asymptotic growth of analytic torsion and torsion cohomology}

Let $\boldsymbol{G}$ be the adjoint group of the unit group of a quaternion algebra $D$ over an imaginary quadratic field $F$. Let $E / F$ be a cyclic Galois extension of odd prime degree $p$.

In this section, we'll prove a torsion cohomology growth theorem for the local systems $\mathcal{L}_{a, b}$ defined in Section 7A over a sequence of locally symmetric spaces for $R_{E / F} \boldsymbol{G}$. The trace formula comparison that we have proven in Section $4 \mathrm{D}$ can be combined with the results of [Bergeron and Venkatesh 2013] to estimate the 
asymptotic growth of torsion in homology through a sequence $\mathcal{M}_{\mathcal{U}_{N}}$ of such spaces where $\mathcal{U}_{N}$ is a parahoric level structure (see Definition 6.16).

We will recall the definition of a strongly acyclic family used in [Bergeron and Venkatesh 2013] in Section 8A. In Section 8B, we will prove a growth theorem for twisted analytic torsion. In Section 8C, we will combine our growth theorem for twisted analytic torsion with the Bismut-Zhang theorem to prove a torsion cohomology growth theorem.

8A. Redux of strong acyclicity. Let $L \rightarrow M$ be a metrized local system over a compact Riemannian manifold $M$. Let $M_{n} \stackrel{\pi_{n}}{\longrightarrow} M$ be a sequence of covers.

Definition 8.1 (strong acyclicity). The family $L_{n}=\pi_{n}^{*} L \rightarrow M_{n}$ is strongly acyclic if the spectra of the Laplace operators $\Delta_{i, L_{n}}, 0 \leq i \leq \operatorname{dim} M$, admit a uniform spectral gap. That is, there is some $\epsilon>0$ such that $\lambda>\epsilon$ for any eigenvalue $\lambda$ of any Laplace operator $\Delta_{i, L_{n}}$.

Bergeron and Venkatesh [2013] crucially use this hypothesis in proving a "limit multiplicity formula for analytic torsion"; it enables them to uniformly control the long time asymptotics of an infinite family of heat kernels. The surprising fact is that strongly acyclic local systems over locally symmetric spaces are abundant.

Let $\boldsymbol{H}$ be a semisimple algebraic group over $F$. Let $M$ be an associated locally symmetric space $\Gamma \backslash \boldsymbol{H}\left(F_{\mathbb{R}}\right) / K$.

Let $\rho: \boldsymbol{H} \rightarrow \mathrm{GL}\left(V_{\rho}\right)$ be a representation over $F$. Any $O_{F}$-lattice inside $V_{\rho}$ which is stable by $\Gamma$ gives rise to a local system $L_{\rho}^{0} \rightarrow M$. We will require the following strengthened version of Theorem 7.1.

Theorem 8.2 [Bergeron and Venkatesh 2013, Theorem 4.1]. Suppose we have $d \rho: \operatorname{Lie}\left(\boldsymbol{H}\left(F_{\mathbb{R}}\right)\right) \rightarrow \operatorname{End}(V) \nsucceq d \rho \circ \theta$. Then for any family of covers $M_{n} \rightarrow M$, the family $L_{n}=\pi_{n}^{*} L \rightarrow M_{n}$ is strongly acyclic.

8A1. Representations of $\mathrm{PGL}_{2}(\mathbb{C})$ and strong acyclicity. Fix a complex embedding $\iota: E \hookrightarrow \mathbb{C}$. There is an isomorphism $\boldsymbol{G}\left(E_{\mathbb{R}}\right) \cong \mathrm{PGL}_{2}(\mathbb{C})^{p}$. Via this identification, $\sigma$ acts by a cyclic shift.

The irreducible representations of $\mathrm{SL}_{2}(\mathbb{C})$ are enumerated as

$$
\rho_{a, b}=V_{a} \otimes \overline{V_{b}}
$$

where $V_{m}=\operatorname{Sym}^{m}\left(\mathbb{C}^{2}\right)$ and where the - denotes conjugation of a complex structure. In order to factor through $\mathrm{SL}_{2}(\mathbb{C}) /\{ \pm 1\}=\mathrm{PGL}_{2}(\mathbb{C})$, we must have $a+b$ even.

Thus, the representations of $\mathrm{PGL}_{2}(\mathbb{C})^{p}$ isomorphic to their $\sigma$-twists are of the form $\tilde{\rho}_{a, b}:=\left(V_{a} \otimes \overline{V_{b}}\right)^{\bigotimes p}$. The representation $\tilde{\rho}_{a, b}$ matches the representation $\rho_{a, b}:=V_{a} \otimes \bar{V}_{b}$ of $\boldsymbol{G}\left(F_{\mathbb{R}}\right) \cong \mathrm{PGL}_{2}(\mathbb{C})$ (see Section $4 \mathrm{C}$ ). 
Remark 8.3. The $\rho_{a, b}$ from above is a slight abuse of notation. After having fixed a complex embedding $\iota$ of $E$, these are the homomorphisms on $F \otimes_{\mathbb{Q}} \mathbb{R}$-valued points induced by $\rho_{a, b}$ of Section 7A.

As explained in [Bergeron and Venkatesh 2013], the representations $\rho_{a, b}$ are strongly acyclic if and only if $a \neq b$. They verify that twisting by the (standard) Cartan involution $\theta$ yields $\rho_{a, b}^{\theta}=\rho_{b, a} ¥ \rho_{a, b}$. By [Bergeron and Venkatesh 2013, Lemma 4.1], this implies that $\rho_{a, b}$ is strongly acyclic.

By the same token, for the Cartan involution $\theta$ of $\mathcal{K}$, we see that

$$
\tilde{\rho}_{a, b}^{\theta}=\tilde{\rho}_{b, a} ¥ \tilde{\rho}_{a, b} \quad \text { for } a \neq b,
$$

implying that the sequence of local systems $\mathcal{L}_{\tilde{\rho}_{a, b, l}} \rightarrow \mathcal{M}_{\mathcal{U}_{N}}$ defined in Section 7A is strongly acyclic. In particular, the rational cohomology of this entire family of local systems vanishes.

\section{B. Growth of twisted analytic torsion.}

Theorem 8.4. Assume that $E / F$ is everywhere tamely ramified. Assume further that the set $\Sigma$ of places where $E / F$ is ramified is disjoint from the set of places where the quaternion algebra $D$ is ramified. Fix a complex embedding $\iota: E \hookrightarrow \mathbb{C}$.

Let $\mathcal{U}_{N} \subset \mathcal{U}_{0}$ denote a sequence of compact open subgroups of $\boldsymbol{G}\left(\mathbb{A}_{E}^{\mathrm{fin}}\right)$ such that:

- The injectivity radius of $\mathcal{M}_{\mathcal{U}_{N}}$ approaches $\infty$.

- The level structures $\mathcal{U}_{N}=\prod_{v} \mathcal{U}_{N, v}$ are globally tamely parahoric.

Let $U_{N, v}$ be a level structure associated with $\mathcal{U}_{N, v}$ (see Definition 5.3) for all places $v \notin \Sigma$ and let $U_{N, v}$ be an Iwahori subgroup of $\operatorname{PGL}_{2}\left(F_{v}\right)$ if $v \in \Sigma$. For the matching local systems $L_{a, b}, \mathcal{L}_{a, b}$ defined in Section $7 A$,

$$
\lim _{N \rightarrow \infty} \frac{\log \tau_{\sigma}\left(\mathcal{M}_{\mathcal{U}_{N}}, \mathcal{L}_{a, b, l}\right)}{\operatorname{vol}\left(M_{U_{N}}\right)} \rightarrow p \cdot(1 / 2)^{n} \cdot c_{a, b} \cdot \prod_{i=1}^{n}\left(2\left|\boldsymbol{B}\left(k_{F_{v}}\right)\right|-1+\frac{1}{q_{v}}\right) \neq 0
$$

where $\boldsymbol{B}$ denotes the corresponding Borel subgroup of $\mathrm{PGL}_{2} / O_{F_{v}}$ and $c_{a, b}$ is a nonzero constant, the $L^{2}$-torsion of $\left(\mathrm{PGL}_{2}(\mathbb{C}), \rho_{a, b, l}\right)$ (see [Bergeron and Venkatesh 2013, Theorem 4.5]).

Proof. Because the level structure $\mathcal{U}_{N}$ is globally tamely parahoric, Theorem 6.17 implies that $\mathbf{1}_{\mathcal{U}} d u$ and $\mathbf{1}_{U^{\Sigma}} d u^{\Sigma} \times \prod_{v \in \Sigma} m_{E_{v} \text {,ur }}$ are matching test functions (see Proposition 6.12 and Theorem 6.15 for a definition of the test function $m_{E_{v} \text {,ur }}$ ). Expand the latter measure as

$$
\mathbf{1}_{U^{\Sigma}} d u^{\Sigma} \times \prod_{v \in \Sigma} m_{E_{v}, \text { ur }}=\sum c_{v_{1}, i_{1}} \cdots c_{v_{n}, i_{n}} \mathbf{1}_{\prod_{j=1}^{n} U_{v_{j}, i_{j}} \times U^{\Sigma}} \prod_{j=1}^{n} d u_{v_{j}, i_{j}} \times d u_{\Sigma}
$$


as in Theorem 6.17. Our abstract matching corollary for analytic torsion (Corollary 4.19) proves that

$$
\log \tau_{\sigma}\left(\mathcal{M}_{\mathcal{U}_{N}}, \mathcal{L}_{a, b, l}\right)=p \sum c_{v_{1}, i_{1}} \cdots c_{v_{n}, i_{n}} \log \tau\left(M_{i_{1}, \ldots, i_{n}}, L_{a, b, l}\right),
$$

where

$$
U_{i_{1}, \ldots, i_{n}}:=\prod_{j=1}^{n} U_{v_{j}, i_{j}} \times U^{\Sigma}, \quad M_{i_{1}, \ldots, i_{n}}=\boldsymbol{G}(F) \backslash \boldsymbol{G}\left(\mathbb{A}_{F}\right) / K U_{i_{1}, \ldots, i_{n}}
$$

(see Theorem 6.17 for further discussion of this notation). For each tuple $\left(i_{1}, \ldots, i_{n}\right)$, the family of local systems $\mathcal{L}_{a, b, \iota} \rightarrow M_{i_{1}, \ldots, i_{n}}$ is strongly acyclic, by the discussion of Section 8A1. Therefore, we may apply the "limit multiplicity theorem for torsion" from [Bergeron and Venkatesh 2013, Theorem 4.5]. Dividing by $\operatorname{vol}\left(M_{U_{N}}\right)$ and taking the limit as $N \rightarrow \infty$, the result follows.

\section{C. Cohomology growth theorem.}

Theorem 8.5. Enforce all the notation and assumptions of Section $8 B$. Let $\mathcal{U}_{N} \subset \mathcal{U}_{0}$ denote a sequence of compact open subgroups of $\boldsymbol{G}\left(\mathbb{A}_{E}^{\mathrm{fin}}\right)$ such that:

- The injectivity radius of $\mathcal{M}_{\mathcal{U}_{N}}$ approaches $\infty$.

- The level structures $\mathcal{U}_{N}=\prod_{v} \mathcal{U}_{N, v}$ are globally tamely parahoric.

- The p-adic part of the cohomology of $\mathcal{L}_{a, b}^{0}$ is controlled as follows, for all $i$ :

$$
\frac{\log \left|H^{i}\left(\mathcal{M}_{\mathcal{U}_{N}}, \mathcal{L}_{a, b}^{0}\right)\left[p^{\infty}\right]\right|}{\operatorname{vol}\left(M_{U_{N}}\right)} \rightarrow 0
$$

- There is not too much mod p cohomology in $\left.\mathcal{L}_{a, b}^{0}\right|_{\mathcal{M}_{\mathcal{U}_{N}}^{\sigma}}$, i.e.,

$$
\frac{\log \left|H^{i}\left(\mathcal{M}_{\mathcal{U}_{N}}^{\sigma}, \mathcal{L}_{a, b, \mathbb{F}_{p}}^{0}\right)\right|}{\operatorname{vol}\left(M_{U_{N}}\right)} \rightarrow 0
$$

Then it follows that, for $a \neq b$,

$$
\frac{\sum^{*} \log \left|H^{i}\left(\mathcal{M}_{\mathcal{U}_{N}}, \mathcal{L}_{a, b}^{0}\right)_{\text {tors }}^{\sigma-1}\right|-\frac{1}{p-1} \log \left|H^{i}\left(\mathcal{M}_{\mathcal{U}_{N}}, \mathcal{L}_{a, b}^{0}\right)_{\text {tors }}^{P(\sigma)}\right|}{\operatorname{vol}\left(M_{U_{N}}\right)} \rightarrow d_{a, b} \neq 0 .
$$

Proof. Fix a complex embedding $\iota: E \hookrightarrow \mathbb{C}$. By Theorem 8.4, there is a limiting identity

$$
\lim _{N \rightarrow \infty} \frac{\log \tau_{\sigma}\left(\mathcal{M}_{\mathcal{U}_{N}}, \mathcal{L}_{a, b, \iota}\right)}{\operatorname{vol}\left(M_{U_{N}}\right)} \rightarrow p \cdot(1 / 2)^{n} \cdot c_{a, b} \cdot \prod_{i=1}^{n}\left(2\left|\boldsymbol{B}\left(k_{F_{v_{i}}}\right)\right|-1+\frac{1}{q_{v_{i}}}\right) \neq 0 .
$$


Combining [Lipnowski 2014, Proposition 4.5] with the results of [Lipnowski 2014, §5], specifically [Lipnowski 2014, Example 5.6], we know that

$$
\tau_{\sigma}\left(\mathcal{M}, \mathcal{L}_{l}\right)=\operatorname{RT}_{\sigma}\left(\mathcal{M}, \mathcal{L}_{l}\right) ;
$$

we have abbreviated $\mathcal{M}:=\mathcal{M}_{\mathcal{U}_{N}}, \mathcal{L}=\mathcal{L}_{a, b}$. Let $A^{\bullet}$ denote the Morse-Smale complex for $\mathcal{L}_{\iota}$ and a fixed invariant weakly gradient-like vector field $X$ on $\mathcal{M}$ satisfying Morse-Smale transversality. As proven in [Lipnowski 2014, Lemma 1.21], there exist $f, f^{\prime} \in E$ such that

$$
\log \mathrm{RT}_{\sigma}\left(A^{\bullet}\right)=\log |\iota(f)|-\frac{1}{p-1} \log \left|\iota\left(f^{\prime}\right)\right|
$$

where

$$
\operatorname{Norm}_{E / \mathbb{Q}}(f)=\prod^{*}\left|H^{i}\left(A^{\bullet}[\sigma-1]\right)\right|, \quad \operatorname{Norm}_{E / \mathbb{Q}}\left(f^{\prime}\right)=\prod^{*}\left|H^{i}\left(A^{\bullet}[P(\sigma)]\right)\right| .
$$

This identity is true for all embeddings $\iota$. Summing (24) over all the embeddings $\iota$, we find that

$\sum_{l} \log \tau_{\sigma}\left(\mathcal{M}, \mathcal{L}_{l}\right)=\sum^{*} \log \left|H^{i}\left(A^{\bullet}[\sigma-1]\right)\right|-\frac{1}{p-1} \sum^{*} \log \left|H^{i}\left(A^{\bullet}[P(\sigma)]\right)\right|$.

By the estimate [Lipnowski 2014, (26) $)_{p}$ combined with [Lipnowski 2014, Proposition 3.7] which relates naive twisted Reidemeister torsion and Reidemeister torsion, we obtain that the right-hand side of (25) is equal to

$$
\begin{aligned}
& -\left(\sum^{*} \log \left|H^{i}\left(\mathcal{M}, \mathcal{L}^{0}\right)\left[p^{-1}\right]^{\sigma-1}\right|-\frac{1}{p-1} \sum^{*} \log \left|H^{i}\left(\mathcal{M}, \mathcal{L}^{0}\right)\left[p^{-1}\right]^{P(\sigma)}\right|\right) \\
& +O\left(\log \left|H^{*}\left(\mathcal{M}, \mathcal{L}^{0}\right)\left[p^{\infty}\right]\right|+\log \left|H^{*}\left(\mathcal{M}, \mathcal{L}_{\mathbb{F}_{p}}^{0}\right)\right|+\log \left|H^{*}\left(\mathcal{M}^{\sigma}, \mathcal{L}_{\mathbb{F}_{p}}^{0}\right)\right|\right) .
\end{aligned}
$$

The remainder big $O$ term in the above equation is $o\left(\operatorname{vol}\left(M_{U_{N}}\right)\right)$ by our assumption on the size of $p$-power torsion in the cohomology $H^{*}\left(\mathcal{M}_{\mathcal{U}_{N}}, \mathcal{L}_{a, b}\right)$ and the $\bmod p$ cohomology $H^{*}\left(\mathcal{M}_{U_{N}}^{\sigma}, \mathcal{L}_{a, b, \mathbb{F}_{p}}\right)$. Dividing both sides of the above equation by $\operatorname{vol}\left(M_{U_{N}}\right)$, applying the limiting identity of (23) separately for each embedding $\iota$, and letting $N \rightarrow \infty$ yields the desired result.

Remark 8.6. One expects the hypothesis

$$
\frac{\log \left|H^{*}\left(\mathcal{M}_{\mathcal{U}_{N}}^{\sigma}, \mathcal{L}_{a, b, \mathbb{F}_{p}}^{0}\right)\right|}{\operatorname{vol}\left(M_{U_{N}}\right)} \rightarrow 0
$$

to hold. For example, Calegari and Emerton [2011, Conjecture 1.2] have conjectured (26) whenever the $M_{U_{N}}$ vary through a $p$-adic analytic tower of hyperbolic 3-manifolds.

Corollary 8.7. Assume that $E / F$ is everywhere tamely ramified of odd prime degree $p$. Assume further that the places where $E / F$ is ramified are disjoint from the places where the quaternion algebra $D$ is ramified. 
Let $\mathcal{U}_{N} \subset \mathcal{U}_{0}$ denote a sequence of compact open subgroups of $\boldsymbol{G}\left(\mathbb{A}_{E}^{\mathrm{fin}}\right)$ such that:

- The injectivity radius of $\mathcal{M}_{\mathcal{U}_{N}}$ approaches $\infty$.

- The level structures $\mathcal{U}_{N}=\prod_{v} \mathcal{U}_{N, v}$ are globally tamely parahoric.

Then it follows that

$$
\underset{N}{\limsup } \frac{\log \left|H^{*}\left(\mathcal{M}_{\mathcal{U}_{N}}, \mathcal{L}_{a, b}^{0}\right)_{\text {tors }}\right|}{\operatorname{vol}\left(\mathcal{M}_{\mathcal{U}_{N}}\right)^{\frac{1}{p}}}>0 \quad \text { for } a \neq b .
$$

Proof. If the $p$-adic part of the cohomology of the sequence $\left(\mathcal{M}_{\mathcal{U}_{N}}, \mathcal{L}_{a, b}^{0}\right)$ is large, i.e., if

$$
\frac{\log \left|H^{*}\left(\mathcal{M}_{\mathcal{U}_{N}}, \mathcal{L}_{a, b}^{0}\right)\left[p^{\infty}\right]\right|}{\operatorname{vol}\left(\mathcal{M}_{\mathcal{U}_{N}}\right)^{\frac{1}{p}}} \nrightarrow 0 \quad \text { or } \quad \frac{\log \left|H^{*}\left(\mathcal{M}_{\mathcal{U}_{N}}, \mathcal{L}_{a, b, \mathbb{F}_{p}}^{0}\right)\right|}{\operatorname{vol}\left(\mathcal{M}_{\mathcal{U}_{N}}\right)^{\frac{1}{p}}} \nrightarrow 0,
$$

then the conclusion follows vacuously.

If the mod $p$ cohomology of $\left(\mathcal{M}_{\mathcal{U}_{N}}^{\sigma}, \mathcal{L}_{a, b, \mathbb{F}_{p}}^{0}\right)$ is large, i.e., if

$$
\frac{\log \left|H^{*}\left(\mathcal{M}_{\mathcal{U}_{N}}^{\sigma}, \mathcal{L}_{a, b, \mathbb{F}_{p}}^{0}\right)\right|}{\operatorname{vol}\left(M_{U_{N}}\right)} \nrightarrow 0,
$$

then the conclusion follows by Smith theory [Bredon 1972, § III]. Indeed [Bredon 1972, § III.4.1],

$$
\operatorname{dim}_{\mathbb{F}_{p}} H^{*}\left(\mathcal{M}_{\mathcal{U}_{N}}^{\sigma}, \mathcal{L}_{a, b, \mathbb{F}_{p}}^{0}\right) \leq \operatorname{dim}_{\mathbb{F}_{p}} H^{*}\left(\mathcal{M}_{\mathcal{U}_{N}}, \mathcal{L}_{a, b, \mathbb{F}_{p}}^{0}\right) .
$$

Since $\left(\mathcal{M}_{U_{N}}, \mathcal{L}_{a, b}^{0}\right)$ has no rational cohomology by Theorem 7.1 , the result follows by the universal coefficient theorem.

Otherwise, all hypotheses of Theorem 8.5 are met and so its more refined conclusion holds. ${ }^{2}$

8D. Comparison to p-adic methods. Let $\boldsymbol{H}$ be a smooth semisimple algebraic group over $\mathbb{Z}_{(p)}$. Let $\mathcal{U}_{n, p}=\operatorname{ker}\left(\boldsymbol{H}\left(\mathbb{Z}_{p}\right) \rightarrow \boldsymbol{H}\left(\mathbb{Z}_{p} / p^{n} \mathbb{Z}_{p}\right)\right)$, let $\mathcal{U}^{p} \subset \boldsymbol{H}\left(\mathbb{A}^{\infty, p}\right)$ be a fixed compact open subgroup, and let $\mathcal{K}_{H} \subset \boldsymbol{H}(\mathbb{R})$ denote a maximal compact subgroup. As a byproduct of their study of completed cohomology, Calegari and Emerton [2009] are able to prove nontrivial upper and lower bounds on cohomology growth for the $p$-adic analytic tower of manifolds $\mathcal{M}_{\mathcal{U}_{n}}=\boldsymbol{H}(\mathbb{Q}) \backslash \boldsymbol{H}(\mathbb{A}) / \mathcal{K}_{H} \mathcal{U}_{n}^{p} \mathcal{U}_{n, p}$. Using Poincaré duality for completed cohomology, they show

$$
\operatorname{dim}_{\mathbb{F}_{p}} H^{*}\left(\mathcal{M}_{\mathcal{U}_{n}}, \mathbb{E}_{p}\right) \gg \operatorname{vol}\left(\mathcal{M}_{\mathcal{U}_{n}}\right)^{1-\alpha}
$$

\footnotetext{
${ }^{2}$ Theorem 8.5 proves that the size of a cohomology group is exponential in $\operatorname{vol}\left(M_{U_{N}}\right)$. But note that $\operatorname{vol}\left(\mathcal{M}_{\mathcal{U}_{N}}\right)^{1 / p}$ and $\operatorname{vol}\left(M_{U_{N}}\right)$ have the same order of magnitude.
} 
where $\alpha=\operatorname{dim}\left(\boldsymbol{H}(\mathbb{R}) / K_{H}\right) / \operatorname{dim} \boldsymbol{H}(\mathbb{R})$; Calegari and Emerton [2011] prove this for $\mathcal{M}_{\mathcal{U}_{n}}$ a tower of hyperbolic 3-manifolds and Calegari [2013] extends this to general $\boldsymbol{H}$. For any local system $\mathcal{L}$ arising from a representation of $\boldsymbol{H}$ defined over $\mathbb{Q}$ with $\mathcal{L}_{\mathbb{Q}}$ acyclic, they deduce that

$$
\log \left|H^{*}\left(\mathcal{M}_{\mathcal{U}_{n}}, \mathcal{L}\right)_{\text {tors }}\right| \geq \log \left|H^{*}\left(\mathcal{M}_{\mathcal{U}_{n}}, \mathcal{L}\right)\left[p^{\infty}\right]\right| \gg \operatorname{vol}\left(\mathcal{M}_{\mathcal{U}_{n}}\right)^{1-\alpha}
$$

as an immediate consequence. It is noteworthy that $\alpha=1 / 2$ if $\boldsymbol{H}(\mathbb{R})$ is a complex Lie group and $1-\alpha \geq 1 / 3$ for arbitrary $\boldsymbol{H}$.

Now let $\boldsymbol{H}$ be equal to $\boldsymbol{G}$, the adjoint group of the unit group of a quaternion algebra $D$ over an imaginary quadratic field $F$ (viewed as a $\mathbb{Q}$-group). Suppose $D$ is split at the odd prime $p$. Let $E / F$ be an everywhere tamely ramified cyclic Galois extension of degree $p$. Let $\mathcal{U}_{n}=\mathcal{U}_{n, p} \mathcal{U}^{p} \subset \boldsymbol{G}\left(\mathbb{A}_{E}^{\mathrm{fin}}\right)$ be any parahoric level structure with $\mathcal{U}_{n, p}$ the full level $p^{n}$ congruence subgroup as above. Let $\mathcal{L}=\mathcal{L}_{a, b}^{0}$ with $a \neq b$. Corollary 8.7 yields $\left|H^{*}\left(\mathcal{M}_{\mathcal{U}_{n}}, \mathcal{L}\right)_{\text {tors }}\right| \gg \operatorname{vol}\left(\mathcal{M}_{\mathcal{U}_{n}}\right)^{1 / p}$ whereas $(28)$ yields the strictly better lower bound $\left|H^{*}\left(\mathcal{M}_{\mathcal{U}_{n}}, \mathcal{L}\right)_{\text {tors }}\right| \gg \operatorname{vol}\left(\mathcal{M}_{\mathcal{U}_{n}}\right)^{1 / 2}$. Nonetheless, the lower bounds obtained by $p$-adic methods do not subsume our main theorems on torsion cohomology.

The origin of the torsion cohomology $H^{*}\left(\mathcal{M}_{\mathcal{U}_{n}}, \mathcal{L}\right)_{\text {tors }}$ detected by $p$-adic methods should be regarded as very distinct from the torsion cohomology detected by the methods of this paper.

Cohomology classes accounted for by (28) are an aggregate of mod $p$ congruences between $(\bmod p)$ automorphic representations of $\boldsymbol{G}_{E}$ of arbitrary level.

On the other hand, the cohomology classes accounted for by Theorem 8.5 and Corollary 8.7 conjecturally arise by base change transfer over $\mathbb{Z}$ (see Section 1 and [Calegari and Venkatesh 2012]). Theorem 7.4 proves a numerical incarnation of base change transfer over $\mathbb{Z}$.

Base change for torsion cohomology leads us to expect that torsion detected in Theorem 8.5 is supported at the same primes as torsion in the cohomology of locally symmetric spaces for $\boldsymbol{G} / F$; computations suggest that the latter primes are large and irregular [Şengün 2011]. On the other hand, torsion detected through (28) is supported at a single prime $p$ and gives no information about the prime-to- $p$ part of torsion cohomology.

Theorem 8.5 and Corollary 8.7 detect torsion cohomology growth for any growing family of manifolds $\mathcal{M}_{\mathcal{U}_{n}}$ defined by parahoric level structures $\mathcal{U}_{n}$, including "horizontally growing families". Suppose $D$ is split outside of a finite set of places $S$. Let $\mathcal{U}_{S}$ be an arbitrary parahoric level structure inside $S$. For any place $v$ outside $S$, let $\mathcal{U}_{v}^{S} \subset \prod_{v^{\prime} \notin S} \mathrm{PGL}_{2}\left(O_{E, v^{\prime}}\right)$ be the product group equal to $\mathrm{PGL}_{2}\left(O_{E, v^{\prime}}\right)$ if $v^{\prime} \neq v$ and the Iwahori subgroup at $v$. Let $\mathcal{U}_{v}=\mathcal{U}_{S} \mathcal{U}_{v}^{S}$. Note that (28) does not give any information concerning torsion cohomology growth for horizontally growing families of manifolds like $\left\{\mathcal{M}_{\mathcal{U}_{v}}\right\}_{v \notin S}$. 


\section{List of symbols}

We compile a list of frequently used notation. The descriptions given are consistent with the most common usage of the symbols. The reader should be warned, however, that within a given chapter or section, the symbols might carry a slightly different meaning; such local changes of notation will be made clear as necessary.

\section{Algebraic groups and representation theory notation.}

- $E / F$ denotes a cyclic Galois extension of number fields of odd prime degree $p$ with Galois group $\Gamma_{E / F}=\langle\sigma\rangle$. The rings $O_{E}, O_{F}$ denote the ring of integers of $E$ and $F$ respectively.

- For a field extension $N / F$, we let $\iota: N \hookrightarrow \mathbb{C}$ denote a complex embedding of $N$ and the induced complex embedding of $F$.

- $D$ denotes a quaternion algebra over $F$.

- $\boldsymbol{H}$ denotes a semisimple algebraic group over a number field $F$.

- $\boldsymbol{G}$ denotes the adjoint group of $\underline{D}^{\times}$.

- $\mathcal{U}$ denotes a compact open Galois-stable subgroup of $\boldsymbol{G}\left(\mathbb{A}_{E}^{\mathrm{fin}}\right)$ or $\boldsymbol{H}\left(\mathbb{A}_{E}^{\mathrm{fin}}\right)$ and $\mathcal{K}$ denotes a Galois-stable maximal compact subgroup of $\boldsymbol{G}\left(E_{\mathbb{R}}\right)$ or $\boldsymbol{H}\left(E_{\mathbb{R}}\right)$.

- $U$ denotes a compact open subgroup of $\boldsymbol{G}\left(\mathbb{A}_{F}^{\mathrm{fin}}\right)$ or $\boldsymbol{H}\left(\mathbb{A}_{F}^{\mathrm{fin}}\right)$ and $K$ denotes a maximal compact subgroup of $\boldsymbol{G}\left(F_{\mathbb{R}}\right)$ or $\boldsymbol{H}\left(F_{\mathbb{R}}\right)$.

- $M_{U}:=\boldsymbol{G}(F) \backslash \boldsymbol{G}\left(\mathbb{A}_{F}\right) / K U$ or $M_{U}:=\boldsymbol{H}(F) \backslash \boldsymbol{H}\left(\mathbb{A}_{F}\right) / K U$.

- $\mathcal{M}_{\mathcal{U}}:=\boldsymbol{G}(E) \backslash \boldsymbol{G}\left(\mathbb{A}_{E}\right) / \mathcal{K U}$ or $\mathcal{M}_{\mathcal{U}}:=\boldsymbol{H}(E) \backslash \boldsymbol{H}\left(\mathbb{A}_{E}\right) / \mathcal{K} \mathcal{U}$.

- $\tilde{\rho}$ is a finite dimensional representation of $R_{E / F} \boldsymbol{G}$ or $R_{E / F} \boldsymbol{H}$ and $\rho$ is a finite dimensional representation of $\boldsymbol{G}$ or $\boldsymbol{H}$.

- $L_{\rho}^{0}$ and $L_{\rho}=L_{\rho}^{0} \otimes_{O_{N}} N$ respectively denote the local system of $O_{N}$-modules and $N$-vector spaces associated to a rational representation $\rho$ in the manner of Section $2 \mathrm{~B}$. $\mathcal{L}_{\tilde{\rho}}^{0}$ and $\mathcal{L}_{\tilde{\rho}}$ denote their equivariant counterparts.

- $\tilde{\pi}$ denotes a representation of $\boldsymbol{G}\left(\mathbb{A}_{E}\right) \rtimes \Gamma_{E / F}$ or $\boldsymbol{H}\left(\mathbb{A}_{E}\right) \rtimes \Gamma_{E / F}$ and $\pi$ denotes a representation of $\boldsymbol{G}\left(\mathbb{A}_{F}\right)$ or $\boldsymbol{H}\left(\mathbb{A}_{F}\right)$.

- $r$ denotes the regular representation of $\boldsymbol{G}\left(\mathbb{A}_{F}\right)$ on $L^{2}\left(\boldsymbol{G}(F) \backslash \boldsymbol{G}\left(\mathbb{A}_{F}\right)\right)$ and $\mathcal{R}$ denotes the regular representation of $\boldsymbol{G}\left(\mathbb{A}_{E}\right) \rtimes \Gamma_{E / F}$ on $L^{2}\left(\boldsymbol{G}(E) \backslash \boldsymbol{G}\left(\mathbb{A}_{E}\right)\right)$.

- For any representation $\left(\pi, W_{\pi}\right)$ of $\boldsymbol{G}\left(\mathbb{A}_{F}\right)$ and any compactly supported smooth measure $f d g$ on $\boldsymbol{G}\left(\mathbb{A}_{F}\right)$, we let $\pi(f d g)$ denote the convolution operator

$$
\int_{\boldsymbol{G}\left(\mathbb{A}_{F}\right)} f(g) \pi(g) d g \circlearrowleft W_{\pi},
$$

and similarly for representations $\tilde{\pi}$ of $\boldsymbol{G}\left(\mathbb{A}_{E}\right)$. 
- For finite dimensional complex representations $\tilde{\rho}$ and $\rho$ of $\boldsymbol{G}\left(F_{\mathbb{R}}\right)$ and $\boldsymbol{G}\left(E_{\mathbb{R}}\right)$ respectively, we define

$$
\begin{array}{ll}
\mathcal{W}:=L^{2}\left(\boldsymbol{G}(E) \backslash \boldsymbol{G}\left(\mathbb{A}_{E}\right)\right), & \mathcal{W}_{\tilde{\rho}}:=L^{2}\left(\boldsymbol{G}(E) \backslash \boldsymbol{G}\left(\mathbb{A}_{E}\right)\right) \otimes \tilde{\rho}, \\
W:=L^{2}\left(\boldsymbol{G}(F) \backslash \boldsymbol{G}\left(\mathbb{A}_{F}\right)\right), & W_{\rho}:=L^{2}\left(\boldsymbol{G}(F) \backslash \boldsymbol{G}\left(\mathbb{A}_{F}\right)\right) \otimes \rho .
\end{array}
$$

- For a representation $\pi$ of a group $H$ and a representation $V$ of $H$, we let $V[\pi]$ denote the $\pi$-isotypic subspace of $H$, i.e., the image of the canonical evaluation map $\operatorname{Hom}_{H}(\pi, V) \otimes \pi \rightarrow V$.

- For a semisimple group $\boldsymbol{H} / \mathbb{R}$ and a maximal compact subgroup $K \subset \boldsymbol{H}(\mathbb{R})$, we let $\theta$ denote the Cartan involution of $\boldsymbol{G}$ associated to $K$; the fixed point set of $\theta$ acting on $\boldsymbol{G}(\mathbb{R})$ equals $K$.

- If $F$ is a local field with ring of integers $O_{F}$ and maximal ideal $\mathfrak{m}_{F}$, we let $k_{F}$ denote the residue field $O_{F} / \mathfrak{m}_{F}$.

- For any finite dimensional representation $A$ of $\langle\sigma\rangle$, we let $\langle A\rangle$ denote $\operatorname{tr}\{\sigma \mid A\}$.

- Let $V$ and $W$ be finite dimensional representations of groups $A, B$. By $V \otimes W$ we mean the external tensor product representation on $V \otimes W$ :

$$
(a, b) \cdot(v \otimes w)=a v \otimes b w .
$$

If $A=B$, we use $V \otimes W$ to denote the internal tensor product representation on the vector space $V \otimes W$ :

$$
a \cdot(v \otimes w)=a v \otimes a w .
$$

\section{Reidemeister torsion notation.}

- $\sum^{*}$ and $\prod^{*}$ respectively denote alternating sum and alternating product.

- $L \rightarrow M$ denotes a local system of projective $O_{F}, F, \mathbb{Q}, \mathbb{Z}, \mathbb{R}$, or $\mathbb{C}$-modules, depending on the context. $\mathcal{L} \rightarrow \mathcal{M}$ denotes a local system equivariant for the action of a finite group $\Gamma$, usually $\Gamma=\langle\sigma\rangle$ with $\sigma^{p}=1$.

- RT $(X, L)$ denotes the Reidemeister torsion of the Morse-Smale complex $\operatorname{MS}(X, L)$ for a vector field $X$, satisfying Morse-Smale transversality, and a local system $L \rightarrow M$, provided the Morse function $f$ and the implicit volume forms are understood [Bismut and Zhang 1992]. $\mathrm{RT}_{\sigma}(\mathcal{X}, \mathcal{L})$ denotes the twisted Reidemeister torsion of the Morse-Smale complex whenever $\mathcal{L} \rightarrow \mathcal{M}$ is a $\langle\sigma\rangle$-equivariant local system. We often suppress the $X, \mathcal{X}$ and denote these by $\mathrm{RT}(M, L)$ and $\operatorname{RT}_{\sigma}(\mathcal{M}, \mathcal{L})$.

- For an $R$-module $A$ which is acted on $R$-linearly by $\langle\sigma\rangle$, we let $A^{\sigma-1}$ be the set $\{a \in A:(\sigma-1) \cdot a=0\}$ and let $A^{P(\sigma)}$ be the set $\{a \in A: P(\sigma) \cdot a=0\}$ where $P(\sigma)$ denotes the $p$-cyclotomic polynomial $P(x)=x^{p-1}+x^{p-2}+\cdots+1$. Sometimes we denote these by $A[\sigma-1]$ and $A[P(\sigma)]$ as well. 
- For an $R$-module $A$ which is acted on $R$-linearly by $\langle\sigma\rangle$, we define $A^{\prime}$ to be the set $A /(A[\sigma-1] \oplus A[P(\sigma)])$. Similarly, if $A^{\bullet}$ is a complex of $R$-modules acted on $R$-linearly by $\langle\sigma\rangle$, we define $A^{\prime \bullet}:=A^{\bullet} /\left(A^{\bullet}[\sigma-1] \oplus A^{\bullet}[P(\sigma)]\right)$.

\section{Acknowledgements}

This paper is an outgrowth of the author's $\mathrm{PhD}$ thesis. It owes its existence to the inspirational work of Bergeron-Venkatesh [2013] and Calegari-Venkatesh [2012].

The author owes thanks to many people: to Jayce Getz, Les Saper, and Mark Stern for their helpful comments on drafts of this paper; to Nicolas Bergeron for many stimulating discussions on torsion growth and twisted endoscopy; to the anonymous referee whose thoughtful feedback greatly improved this paper.

Last but not least, the author would like to express his deep gratitude to his advisor, Akshay Venkatesh, for sharing so many of his ideas and for providing constant encouragement and support during the preparation of this work.

\section{References}

[Arthur and Clozel 1989] J. Arthur and L. Clozel, Simple algebras, base change, and the advanced theory of the trace formula, Annals of Mathematics Studies 120, Princeton University Press, 1989. MR 90m:22041 Zbl 0682.10022

[Bergeron and Venkatesh 2013] N. Bergeron and A. Venkatesh, "The asymptotic growth of torsion homology for arithmetic groups”, J. Inst. Math. Jussieu 12:2 (2013), 391-447. MR 3028790 Zbl 1266.22013

[Bismut and Zhang 1992] J.-M. Bismut and W. Zhang, An extension of a theorem by Cheeger and Müller, Astérisque 205, Société Mathématique de France, Paris, 1992. MR 93j:58138 Zbl 0781.58039

[Bismut and Zhang 1994] J.-M. Bismut and W. Zhang, "Milnor and Ray-Singer metrics on the equivariant determinant of a flat vector bundle", Geom. Funct. Anal. 4:2 (1994), 136-212. MR 96f:58179 Zbl 0830.58030

[Borel and Wallach 2000] A. Borel and N. Wallach, Continuous cohomology, discrete subgroups, and representations of reductive groups, 2nd ed., Mathematical Surveys and Monographs 67, American Mathematical Society, Providence, RI, 2000. MR 2000j:22015 Zbl 0980.22015

[Bredon 1972] G. E. Bredon, Introduction to compact transformation groups, Pure and Applied Mathematics 46, Academic Press, New York-London, 1972. MR 54 \#1265 Zbl 0246.57017

[Bushnell and Henniart 2006] C. J. Bushnell and G. Henniart, The local Langlands conjecture for GL(2), Grundlehren der Mathematischen Wissenschaften [Fundamental Principles of Mathematical Sciences] 335, Springer, Berlin, 2006. MR 2007m:22013 Zbl 1100.11041

[Buzzard and Gee 2015] K. Buzzard and T. Gee, "The conjectural connections between automorphic representations and Galois representations”, preprint, 2015. arXiv 1009.0785

[Calegari 2013] F. Calegari, "Torsion in the cohomology of co-compact arithmetic lattices", blog entry, 2013, available at http://galoisrepresentations.wordpress.com/2013/02/06/torsion-in-the-cohomology. Published online February 6, 2013.

[Calegari and Emerton 2009] F. Calegari and M. Emerton, "Bounds for multiplicities of unitary representations of cohomological type in spaces of cusp forms", Ann. of Math. (2) 170:3 (2009), 1437-1446. MR 2011c:22032 Zbl 1195.22015 
[Calegari and Emerton 2011] F. Calegari and M. Emerton, "Mod- $p$ cohomology growth in $p$-adic analytic towers of 3-manifolds", Groups Geom. Dyn. 5:2 (2011), 355-366. MR 2012b:22010 Zbl 1242.57014

[Calegari and Venkatesh 2012] F. Calegari and A. Venkatesh, "A torsion Jacquet-Langlands correspondence", preprint, 2012. arXiv 1212.3847

[Cheeger 1979] J. Cheeger, "Analytic torsion and the heat equation", Ann. of Math. (2) 109:2 (1979), 259-322. MR 80j:58065a Zbl 0412.58026

[Fung 2002] M. G. G. Fung, "Twisted torsion on compact hyperbolic spaces: a representationtheoretic approach”, Asian J. Math. 6:1 (2002), 169-193. MR 2003g:58050 Zbl 1018.58023

[Gelbart and Jacquet 1979] S. Gelbart and H. Jacquet, "Forms of GL(2) from the analytic point of view", pp. 213-251 in Automorphic forms, representations and L-functions, Part 1 (Oregon State University, Corvallis, OR, 1977), edited by A. Borel and W. Casselman, Proc. Sympos. Pure Math. XXXIII, American Mathematical Society, Providence, RI, 1979. MR 81e:10024 Zbl 0409.22013

[Kottwitz 1982] R. E. Kottwitz, "Rational conjugacy classes in reductive groups", Duke Math. J. 49:4 (1982), 785-806. MR 84k:20020 Zbl 0506.20017

[Kottwitz 1986a] R. E. Kottwitz, "Base change for unit elements of Hecke algebras", Compositio Math. 60:2 (1986), 237-250. MR 88e:11039

[Kottwitz 1986b] R. E. Kottwitz, "Stable trace formula: elliptic singular terms", Math. Ann. 275:3 (1986), 365-399. MR 88d:22027 Zbl 0577.10028

[Labesse and Waldspurger 2013] J.-P. Labesse and J.-L. Waldspurger, La formule des traces tordue d'après le Friday Morning Seminar, CRM Monograph Series 31, American Mathematical Society, Providence, RI, 2013. MR 3026269 Zbl 1272.11070

[Langlands 1980] R. P. Langlands, Base change for GL(2), Annals of Mathematics Studies 96, Princeton University Press, University of Tokyo Press, 1980. MR 82a:10032 Zbl 0444.22007

[Lipnowski 2014] M. Lipnowski, “The equivariant Cheeger-Müller theorem on locally symmetric spaces”, J. Inst. Math. Jussieu (online publication October 2014), 1-38.

[Lück 1993] W. Lück, "Analytic and topological torsion for manifolds with boundary and symmetry", J. Differential Geom. 37:2 (1993), 263-322. MR 94e:57054 Zbl 0792.53025

[Müller 1978] W. Müller, "Analytic torsion and R-torsion of Riemannian manifolds", Adv. in Math. 28:3 (1978), 233-305. MR 80j:58065b Zbl 0395.57011

[Müller 1993] W. Müller, "Analytic torsion and $R$-torsion for unimodular representations", J. Amer. Math. Soc. 6:3 (1993), 721-753. MR 93m:58119 Zbl 0789.58071

[Ray and Singer 1971] D. B. Ray and I. M. Singer, " $R$-torsion and the Laplacian on Riemannian manifolds", Advances in Math. 7 (1971), 145-210. MR 45 \#4447 Zbl 0239.58014

[Şengün 2011] M. H. Şengün, “On the integral cohomology of Bianchi groups”, Exp. Math. 20:4 (2011), 487-505. MR 2859903 Zbl 1269.22007

[Speh 1994] B. Speh, "Analytic torsion and automorphic forms", pp. 147-156 in Noncompact Lie groups and some of their applications (San Antonio, TX, 1993), edited by E. A. Tanner and R. Wilson, NATO Adv. Sci. Inst. Ser. C Math. Phys. Sci. 429, Kluwer Acad. Publ., Dordrecht, 1994. MR 96f:58183 Zbl 0845.57028

Communicated by Philippe Michel

Received 2014-05-13 Revised 2015-07-21 Accepted 2015-10-06

malipnow@math.duke.edu Mathematics Department, Duke University, Duke University, Box 90320, Durham, NC 27708-0320, United States 


\section{Algebra \& Number Theory}

msp.org/ant

\section{EDITORS}

MANAGING EDITOR

Bjorn Poonen

Massachusetts Institute of Technology

Cambridge, USA

\author{
EDITORIAL BOARD CHAIR \\ David Eisenbud \\ University of California \\ Berkeley, USA
}

BOARD OF EDITORS

Georgia Benkart

Dave Benson

Richard E. Borcherds

John H. Coates

J-L. Colliot-Thélène

Brian D. Conrad

Hélène Esnault

Hubert Flenner

Sergey Fomin

Edward Frenkel

Andrew Granville

Joseph Gubeladze

Roger Heath-Brown

Craig Huneke

Kiran S. Kedlaya

János Kollár

Yuri Manin

Philippe Michel
University of Wisconsin, Madison, USA

University of Aberdeen, Scotland

University of California, Berkeley, USA

University of Cambridge, UK

CNRS, Université Paris-Sud, France

Stanford University, USA

Freie Universität Berlin, Germany

Ruhr-Universität, Germany

University of Michigan, USA

University of California, Berkeley, USA

Université de Montréal, Canada

San Francisco State University, USA

Oxford University, UK

University of Virginia, USA

Univ. of California, San Diego, USA

Princeton University, USA

Northwestern University, USA

École Polytechnique Fédérale de Lausanne
Susan Montgomery

Shigefumi Mori

Raman Parimala

Jonathan Pila

Anand Pillay

Victor Reiner

Peter Sarnak

Joseph H. Silverman

Michael Singer

Vasudevan Srinivas

J. Toby Stafford

Ravi Vakil

Michel van den Bergh

Marie-France Vignéras

Kei-Ichi Watanabe

Efim Zelmanov

Shou-Wu Zhang
University of Southern California, USA

RIMS, Kyoto University, Japan

Emory University, USA

University of Oxford, UK

University of Notre Dame, USA

University of Minnesota, USA

Princeton University, USA

Brown University, USA

North Carolina State University, USA

Tata Inst. of Fund. Research, India

University of Michigan, USA

Stanford University, USA

Hasselt University, Belgium

Université Paris VII, France

Nihon University, Japan

University of California, San Diego, USA

Princeton University, USA

\section{PRODUCTION}

production@msp.org

Silvio Levy, Scientific Editor

See inside back cover or msp.org/ant for submission instructions.

The subscription price for 2015 is US $\$ 255 /$ year for the electronic version, and $\$ 440 /$ year ( $+\$ 55$, if shipping outside the US) for print and electronic. Subscriptions, requests for back issues and changes of subscribers address should be sent to MSP.

Algebra \& Number Theory (ISSN 1944-7833 electronic, 1937-0652 printed) at Mathematical Sciences Publishers, 798 Evans Hall \#3840, c/o University of California, Berkeley, CA 94720-3840 is published continuously online. Periodical rate postage paid at Berkeley, CA 94704, and additional mailing offices.

ANT peer review and production are managed by EditFLOW ${ }^{\circledR}$ from MSP.

\section{PUBLISHED BY}

- mathematical sciences publishers

nonprofit scientific publishing

http://msp.org/

(C) 2015 Mathematical Sciences Publishers 


\section{Algebra \& Number Theory}

Volume $9 \quad$ No. $10 \quad 2015$

Equivariant torsion and base change

MICHAEL LIPNOWSKI

Induction parabolique et $(\varphi, \Gamma)$-modules

CHRISTOPHE BREUIL

On the normalized arithmetic Hilbert function

MOUNIR HAJLI

SUNE PRECHT REEH

XUANCHENG SHAO

Bounds for Serre's open image theorem for elliptic curves over number fields

DAVIDE LOMBARDO

On 0-cycles with modulus

AMALENDU KRISHNA 